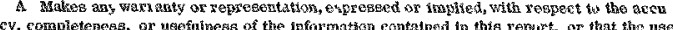

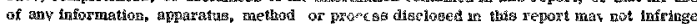

priv atelv awped rights, or

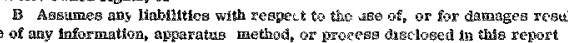

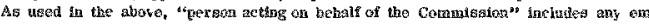

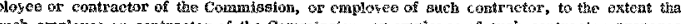
allecemis

ARGONNE NATIONAL LABORATORY

P. O. Box 299

Lemont, Illinois

INTERPRETATION OF COUNTING DATA

by

Ellis P. Steinberg

CHEMISTRY DIVISION

September 26,1956

Operated by The University of Chicago

under

Contract $W-31-109-e n g-38$ 


\section{DISCLAIMER}

This report was prepared as an account of work sponsored by an agency of the United States Government. Neither the United States Government nor any agency Thereof, nor any of their employees, makes any warranty, express or implied, or assumes any legal liability or responsibility for the accuracy, completeness, or usefulness of any information, apparatus, product, or process disclosed, or represents that its use would not infringe privately owned rights. Reference herein to any specific commercial product, process, or service by trade name, trademark, manufacturer, or otherwise does not necessarily constitute or imply its endorsement, recommendation, or favoring by the United States Government or any agency thereof. The views and opinions of authors expressed herein do not necessarily state or reflect those of the United States Government or any agency thereof. 


\section{DISCLAIMER}

Portions of this document may be illegible in electronic image products. Images are produced from the best available original document. 
PREFACE .......................... 3

INTRODUCTION .........................

GENERAL CONSIDERATIONS ................ 5

BETA COUNTING....................... 8

End-Window Counters .................. 8

Detection Probability.................... 9

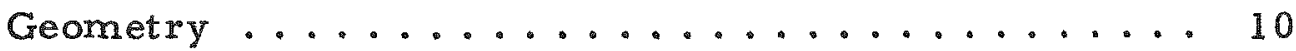

$\mathrm{f}_{\mathrm{W}}$ (factor for air and counter-window absorption) .... 11

$f_{A}$ (factor for scattering effect of air) ........... 14

$f_{C}$ (factor for effect of sample covering) ........ 14

$f_{B}$ (factor for effect of backscattering) ......... 15

$f_{\mathrm{H}}$ (factor for the effect of the housing) ......... 16

$f_{S}$ (self-absorption and self-scattering factor) ..... 18

Suggested Techniques for End Window Counting ........ 20

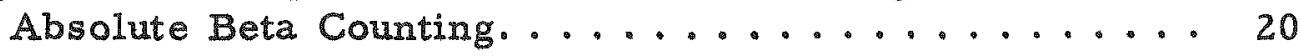

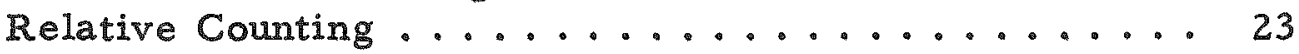

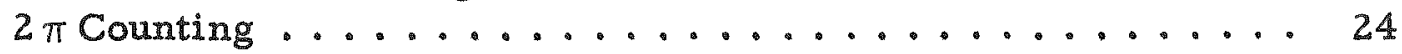

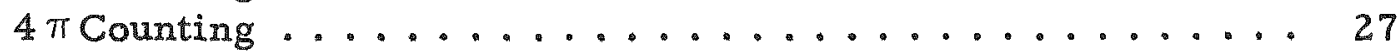

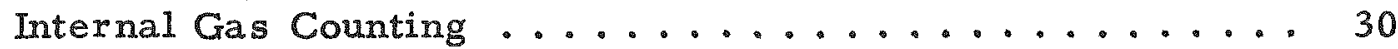

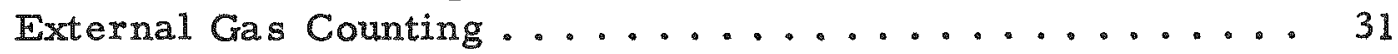

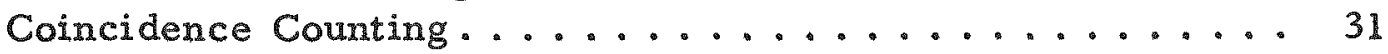

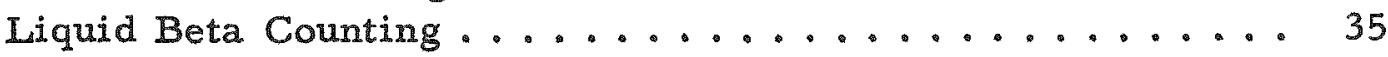

Beta Scintillation Counting ............... 35

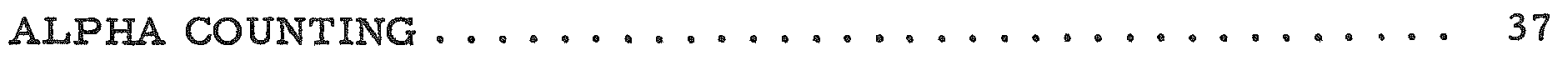

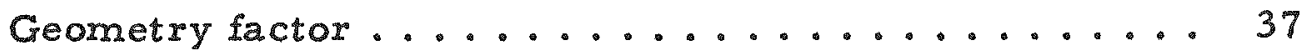

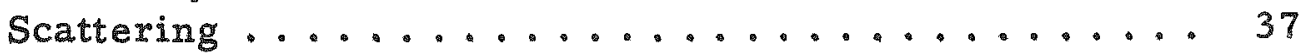

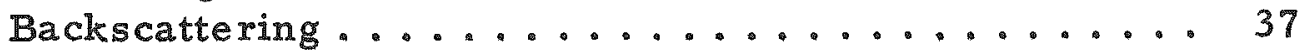

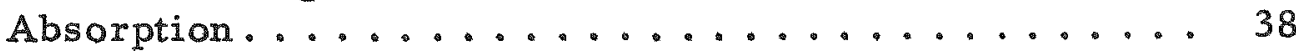

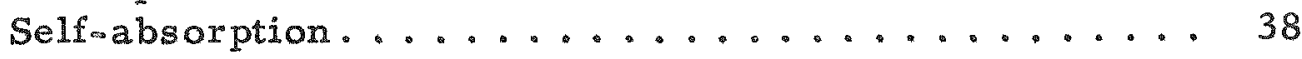

Plateaus ....................... 40

Beta pulse pile-up................. 40

Counting standards ..................... 40

Resolution losses ................... 41

Absolute counting .................. 41

Relative counting.................. 41

GAMMA COUNTING. ................... 42

Relative Counting .................. 42

Absolute Gamma Counting................... 42

Scintillation Counting - Pulse Height Analysis ....... 43 
TABLE OF CONTENTS

Page

ACKNOWLEDGMENT .................... 51

REFERENCES IN TEXT .................... 52

GENERAL REFERENCES ON THE MEASUREMENT OF

RADIOACTIVITY .......................... 59

APPENDIX I. FORMULAS FOR GEOMETRY CALCULATIONS . . . 61

APPENDIX II. RESOLUTION LOSS CORRECTIONS IN COUNTING . . 63 


\section{PREFACE}

This report was prepared as a contribution to a "Handbook of Instruments and Techniques" to be published by the Subcommittee of Instruments and Techniques of the National Research Council Committee on Nuclear Science. Comments or criticisms would be greatly appreciated by the author. 


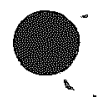

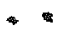

$\because$

$-$

$\because$

.

.

$\therefore$

. .

-

$\cdot$
$\therefore$
$\therefore$
$\therefore$

.

$\because$

$-\triangle$

(3)

- 


\title{
INTERPRETATION OF COUNTING DATA
}

\section{by}

\author{
Ellis P. Steinberg
}

\section{INTRODUCTION}

\begin{abstract}
The general availability of radioisotopes has placed a powerful tool in the hands of investigators in many fields of science, and it is the purpose of this report to aid these workers in the design of meaningful experiments utilizing radioactivity. An understanding of the various factors which may contribute to errors in measurement is essential for the choice of appropriate techniques and measuring devices and for the proper evaluation of experimental data. This report is not intended as a treatise on the measurement of radioactivity, but it is hoped that the presentation of a handy guide to some of the problems involved and the techniques generally employed in avoiding or correcting for them, together with references to the pertinent literature, will provide an a dequate orientation and introduction to the subject.
\end{abstract}

\section{GENERAL CONSIDERATIONS}

Although the discussion here will be limited to counting methods of radioactive assay, which are of more general interest, a variety of techniques are available, e.g., photographic, calorimetric, charge collection, colloid deposition, crystal coloring, etc. Different techniques may, of course, involve somewhat different error analyses; however, the problems associated with the determination of the absolute disintegration rate of a radioactive source by counting techniques afford an excellent example of the commonly encountered difficulties. Such a determination involves a knowledge of the decay scheme of the radioactive nuclide and the efficiency of the detector for the particles or rays emitted by the source. It is thus necessary to know the fraction of the radiation leaving the source which enters the sensitive volume of the detector ("geometry factor") and the intrinsic efficiency of the detector for the radiation. The interactions of radiation with matter alter the simple geometrical relationship between the source and detector by processes of absorption and scattering in the material of the sample itself, its support, any covering material, the space between the sample and the counter, the counter window, and the housing of the detector unit. These effects are complex and depend critically on the size and material of the sample and its support and on the energy and type of the radiation. Special techniques are required to minimize these effects for the determination of disintegration rates to a few percent accuracy. 
For many purposes an experiment can be designed so that relative counting rates of samples of a particular nuclide will suffice. It is then only necessary to count under nearly identical conditions to obtain precisions of the order of one to two percent. This criterion, in practice, is relatively simple to meet and, whenever possible, comparative counting is therefore recommended. Of course, if it is necessary to compare the activity of sources which differ in chemical or physical nature or in the energy of their radiations, these differences must be taken into account.

Much time and effort may be saved by the judicious application of published correction factors for the individual and combined effects of backscatter, sample thicknesses, etc., on counting determinations. How ever, it is extremely important to be aware of the sensitivity of the effects to experimental conditions. Where high accuracy is desired it is usually advisable for each investigator to determine the correction for his own conditions or employ techniques which minimize the effects.

In some cases the radioactivity of the nuclide of interest may be complicated by the presence of isotopic or genetically related species which are also radioactive. Corrections for the presence of the se may be made, in general, by taking advantage of the differences in the half-lives, energies, and, perhaps, types of radiation involved. Even in the case of a pure nuclide, different types of radiation may be emitted $\left(e_{.}, \beta^{*}, e^{-m}, X^{m}\right.$, or $\gamma$ rays) and their contributions to the observed counting rates must be evaluated.

Any detector will record counts, even in the absence of a sample, due to the presence of cosmic rays and of ionizing radiation in the materials of construction and in the laboratory air (thoron, radon, etc.). This background rate may vary with laboratory conditions and must be determined under conditions duplicating those under which the sample is counted. It must be subtracted from the sample counting rate. Since the background rate limits the sensitivity of the detector, it is advisable to reduce it to a level which is low in comparison with sample counting rates. The background rate is dependent on the size of the detector. For standard end-window Geiger and proportional counters (about 11" diameter $x 2$ "long) backgrounds of 30 to 50 counts per minute are common. This rate may be reduced by a factor of two to three by the common practice of enclosing the detector in a lead or iron housing of one to two inches in thickness.

Further shielding from penetrating cosmic radiation may be achieved by surrounding the detector with a ring of additional counters connected so that the detector will not record a count which is coincident with one in the protective ring. The entire assembly is enclosed in an iron, lead, or mercury shield. Such an arrangement - referred to as anti-coincidence shielding(1) - may reduce the background rate to as low as 1 to 2 counts per 
minute. Additional corrections may be necessary for the variation in efficiency of the detector with time (as determined by the routing counting of a suitable standard) and for the finite resolving time of the detector (see Appendix II).

Radioactive decay is a random process and inherent fluctuations in the disintegration rate of any source limit the ultimate accuracy of count= ing data. These fluctuations follow well-known statistical laws and the statistical accuracy of a set of data can be readily calculated. 


\section{BETA COUNTING}

End-Window Counters

Although many specially designed counters are employed to satisfy particular needs, (102) the end-window Geiger counter has probably been the most universally used detector for beta radiation. Consequently, much of the experimental work on the problems of beta counting has been carried out with this detector. Although the Geiger counter still finds useful application, it is generally being replaced with the flow-type, end-window proportional counter. The latter has many advantages. Since it is operated at atmospheric pressure, a thin window (usually 0.5 to $1.0 \mathrm{mg} / \mathrm{cm}^{2}$ plastic foil * coated with a conducting film of Aquadag or evaporated metal) can be used, and hence the range of application can be extended to lower energy betas, while retaining the convenience of counting samples externally. Geiger counters are usually operated at a pressure of ca. $10 \mathrm{~cm}$ of $\mathrm{Hg}$, and window thicknesses greater than about $2 \mathrm{mg} / \mathrm{cm}^{2}$ are most usual. Moreover, the proportional counter permits a much wider range of source strengths to be used since the dead time losses are of the order of $1 \%$ per $10^{5} \mathrm{c} / \mathrm{m}$ com pared with losses of about $0.5 \%$ per $10^{3} \mathrm{c} / \mathrm{m}$ in a Geiger counter. Proportional counters require associated electronic equipment of somewhat higher caliber than Geiger counters (e.g., stable high voltage supplies and nonoverloading amplifiers), but these are readily available. Considerable experience with proportional counters has proved them to have long lifetimes and excellent stability.

Fortunately, the size and shape, as well as the commonly employed holder and shelf arrangement, are very nearly identical for the Geiger and end-window proportional counters, and the extensive studies of various ef fects in beta counting carried out with Geiger counters are in most cases directly applicable to the proportional counter. Of course, there will be differences in some cases where the window thickness plays a significant role, but even here the effects will be qualitatively the same. It cannot be overemphasized that the greatest contribution of the many studies of beta counting reported in the literature lies in their elucidation of the complex effects encountered and presentation of methods of correction. The quantitative data presented are useful for approximate corrections and general orientation. However, for the highest accuracy, the quantitative factors should be determined for the particular experimental arrangement being used.

*For example, rubber hydrochloride, available from Originality House, Inc., 480 Avalon Ave., Akron, Ohio or Mylar (polyethylene terephthalate), available from E. I. DuPont de Nemours \& Co., Wilmington, Delaware. 
The importance of absorption and scattering phenomena in the detection of beta particles was pointed out as early as 1910 by Kovarik and others. (2) Many aspects of the problem of the determination of absolute disintegration rates from counting data have since been investigated experimentally. The complexity of the problem has prevented successful theoretical development, and the magnitude of the effects must usually be determined from empirical data. Although it is not always necessary to obtain absolute disintegration rates, the investigator should be aware of the factors which may contribute to errors in counting determinations.

When particle emission accompanies each decay, a counting yield, $Y$, may be defined(3) as the ratio of the net counting rate observed (corrected for resolution losses and background activity), and the true disintegration rate. Thus

$$
Y=\frac{\text { counts per minute }(\mathrm{c} / \mathrm{m})}{\text { disintegrations per minute }(\mathrm{d} / \mathrm{m})} .
$$

For simplicity, the present discussion will be limited to the case of a simple beta-emitter (such as $\mathrm{P}^{32}$ or $\mathrm{C}^{14}$ ). More complex decay schemes with $\gamma$ rays, $X$ rays and conversion electrons, as well as beta branching, will be discussed later. Although the factors which enter into the determination of $Y$ are complex and not easily separable experimentally, they can, in principle, be treated independently. Then, $(3,4)$

$$
\mathrm{Y}=\frac{\mathrm{c} / \mathrm{m}}{\mathrm{d} / \mathrm{m}}=(\mathrm{I})(\mathrm{G}) \mathrm{f}_{\mathrm{W}} \mathrm{f}_{\mathrm{A}} \mathrm{f}_{\mathrm{C}} \mathrm{f}_{\mathrm{B}} \mathrm{f}_{\mathrm{H}} \mathfrak{f}_{\mathrm{S}}
$$

where $I$ = detection probability, $G=$ geometry, $f_{W}=$ factor for the absorption effect of the counter window and air between source and window, $f_{A}=$ factor for the scattering effect of the air, $f_{C}=$ factor for the effect of any covering material over the sample, $f_{B}=$ factor for the effect of backscattering by the material supporting the source, $f_{H}=$ factor for the effect of the sample and tube-supporting structure and the walls of the housing or shield in scattering counts into the counter, and $f_{S}=$ factor for the effect of the finite mass of the source ("self-absorption" and "self-scattering").

Detection Probability. Ideally, every particle which passes through the window of an end-window Geiger or proportional counter will form at least one ion-pair, initiate a Townsend avalanche, and register a count. Actually, however, there is a finite probability that an ion-pair will not be formed. This probability may be calculated(4) and is dependent on the specific primary ionization constant of the counter gas, the pressure of the gas, the energy, and the path length of the particle through the volume of the counter. In general, the detection probability is close to unity and no corrections are required except for work of extremely high accuracy. For cases of high geometry and energetic betas, the path length obliquely through the 
corners of the counter may be too short to produce sufficient ionization to register a pulse. This will result in a shift of the knee of the voltage plateau to higher values. Defining apertures in front of the window may counteract this effect by collimating the particles, but these may also introduce scattering effects from the edges. It may be more advisable to reduce the geometry of counting by increasing the source to tube distance if the added path length does not introduce an absorption problem.

Effects such as these illustrate the care that should be exercised in the choice of operating voltage. Voltages just above the plateau knee for weak betas may be below the plateau for energetic betas, since the specific ionization of electrons decreases with increasing energy. On the other hand, the resolving time of the counter increases with increasing voltage along the plateau, and a voltage which insures a high detection probability may introduce higher resolution losses.

In practice this problem is generally met by determining the geometry of the source position with standards having approximately the same energy and counting rate as the sources to be measured. In this case, a "geometric efficiency" is actually determined, in which the detection probability and resolution losses cancel.

Geometry. Radiation is emitted isotropically from a point source and, in the absence of absorption and scattering phenomena, the fraction of the solid angle of $4 \pi$ radians subtended by the sensitive volume of the coun ter at the source will represent the fraction of the emitted radiation which is counted. This fraction is known as the "geometry" or "physical geometry" and is determined simply by the dimensions of the system. Formulas may also be derived for the geometry of extended sources. These are given in Appendix $I_{\text {. }}$

The geometry is generally determined by counting standards of known disintegration rates (determined by coincidence counting, $4 \pi$ counting, or other techniques) in the desired position. In order to minimize the cor rections for absorption and scattering, standards are prepared in thin, uniform deposits on thin backings. $(4,5,6,59 \mathrm{e})$ The ratio of the counting rate of the standard (suitably corrected for absorption and scattering effects, as well as background activity and any resolution losses) and the known disintegration rate gives the geometric efficiency.

It has been reported $(4,5,7)$ that the observed geometric efficiency is slightly lower than that calculated from the dimensions of the counting sys tem. This has been interpreted as indicating that the active volume of the counter begins a few millimeters behind the window. A "defined geometry" technique employing a brass collimator has been suggested $(7,8,9)$ to avoid this difficulty. However, Fried, et al. (10) observed significant increases 
In the curvature at abscrption curves $C^{60}$ and $R$ aE di tow absorber thicknt sses and demonstrated that when apprepriatc corrections for absorption ir. the windw ard gas path app apd the obser t and cavcutated geome tries do. Indetd ager whith the acuracy of the measurements.

The ge imetric etficiency Ic $x$ extended sowres has been exaluated tmpirica 2 ard show to dgree we. wh the caiculated gecmetry. $(1,11-14)$

Athough, as pointed cut abore, it is desirabe to use standards hatrg the same treygy as the sources cf lnterest, sycus in the extrapola tion ct absorptur curves (see section on $f_{\text {W }}$ ) tc zero thickness may result trom diufererces in the beta spectra shapes uf two nucxides having the same maximum beta 4 rgy. Manov(15) suggests that many standards may be requized le the accutate calsbration of sources of gencral interestand has rerifwed the status of the work on the standardization of radroactive soarces to 1954

I $W$ (factor fur air and counter window absorption). Since beta particles are easily scattered in passing through matter an absurbing material placed between the source and detector mavreduce the intersity of radiation reaching the countex by true absorption or by scattering particies out of thes norma paths toward the detector. On the other hand some paricles may be scattex into the derector, increasing the apparent intensity. These effects ax cyicaly deperdent on the Ir atr positions of souxce, absorber. and detector. $(4.16-19)$ if the absorbing mateida. is cuose the counter window scattering effects are minimized, and true absorption effects are observed The fractional reduction in counting rate dut to the window and air $f_{W}$ w. be depcridert on the energy $c$ the ydiation and the thickness of the wrdow and air path The factor. "t $W$, is determind from a absorption curve b, extrapolation to a negative id u of added absorber ccrresponding

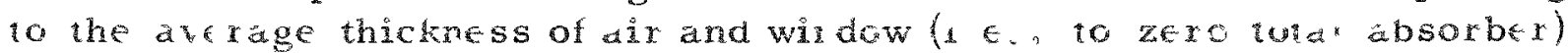
(see Figure 1) (In determining such a curve. the absorbing fois showd a way be paced close to the cowner window Since the arerage atomic number of alr and prastic countex window materials are c cse to that of the convenient y prepared aluminum absorbers. the latter is usuany used for absorbing foins) The fart that the air is an extended absoxber and contributes scattering is reguected herte but 1 s taken into account in the factor $f_{A}$ discussrd be ow

- It is convenient to express the "thickess of asorbirg materiat in terms of surface densit, (mass per writ ar a), since on this basis the absorption of beta parile 1 es near" irdeperdent of the rature ot the materza" particualu tur absoxbers of low atcmic number 


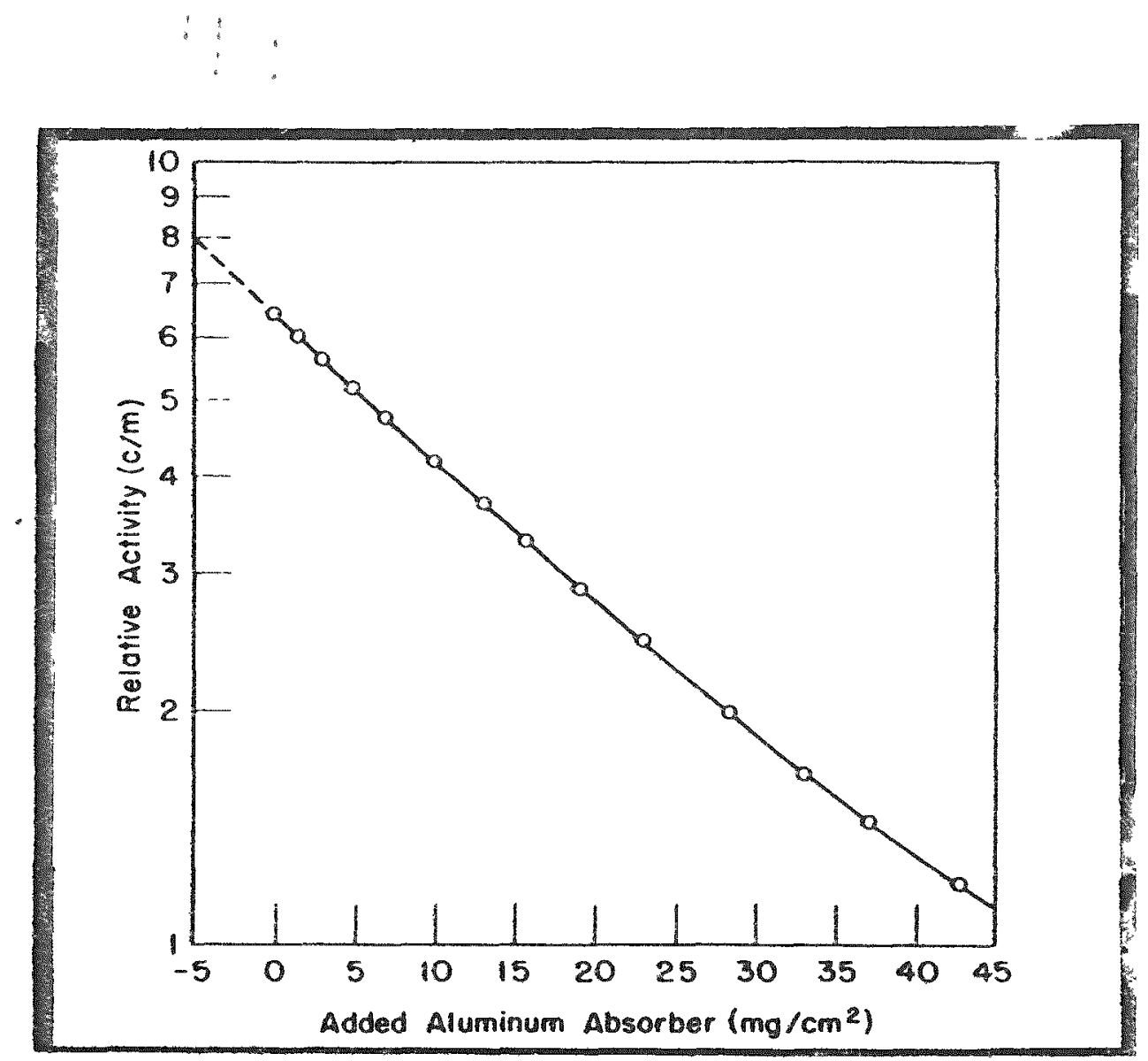

Figure 1

illustration of extrapolation of initial portion of a typical absorption curve to correct for $5.0 \mathrm{mg} / \mathrm{cm}^{2}$ absorption in tube window and air path. (Factor $f_{W}$ )

Serious errors may axise in the exirapolation of absorption curves to aero total absorber. It is often stated that the absorption of beta particles is nearly exponential. This statement can be misleading. The complex nature of beta spectra and absorption and scattering phenomena do, in many cases, give rise to absorption curves which are esponential over the initial portions. In fact, initial half-thickness values or absorption coefficients are conveniently used to determine corrections for absorption andytically (i.e., $N=N_{o} e^{-\lambda t}$ ). (For approximate alculations a curve of initial half-thickness versus energy may be useful and is given in Figure 2.) Careful determinations of absorption curves, however, particularly using the thin window proportional counters, have indicated that the exponential character of beta absorption is not a valid generalization, ard the absorption curves of many nuclides indicate initial curvatures. Zumali(4) has suggested a formula for calculating the extrapolated counting rate in terms of the initial slope of the absorption curve and the curvature. In determining the factor $f_{1}$ graphically it is obviously necessary to obtain a sufficient number of points on an 


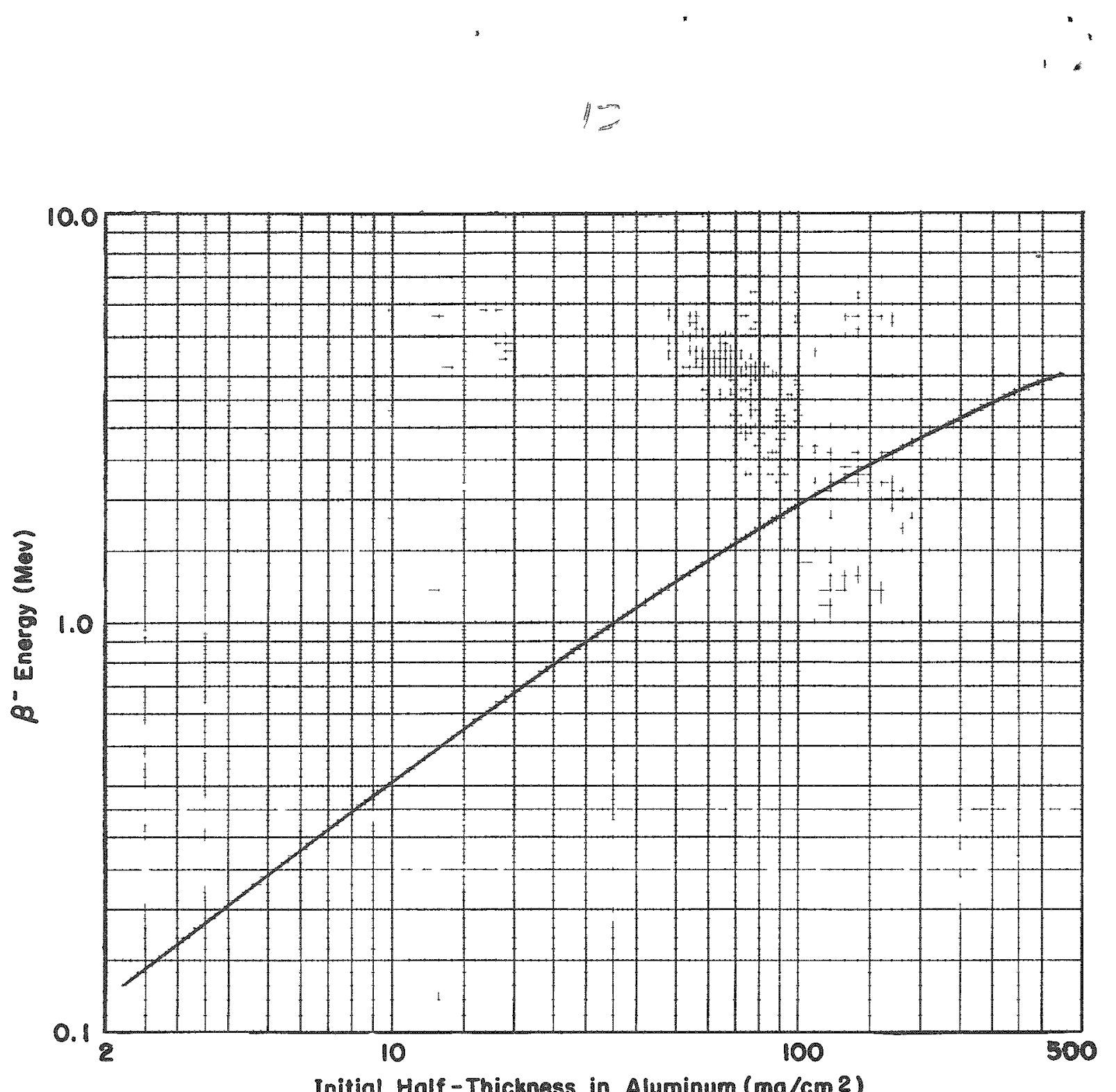

Initial Half-Thickness in Aluminum (mg/cm 2 )

Figure 2

Energv versus half-thickness in aluminum for $\hat{\beta}$-particles. (These data were obtained for samples weighing about $20 \mathrm{mg}$ and mounted over a $2 \mathrm{~cm}^{2}$ area on filter paper and covered with $3 \mathrm{mg} / \mathrm{cm} \mathrm{c}^{2} \mathrm{cel}-$ lophane. They were counted on the first shelf (geometry factor $=25 \%$ of $4 \pi$ ) of a standard Geiger counter (tube window $\left.\left.=3.0 \mathrm{mg} / \mathrm{cm}^{2}\right).\right)$ 
absorption curve as close to zero absorber as possible in order to increase the accuracy of the extrapolation. Since the absorption is nearly exponential in many cases, it is convenient to use semi-log paper for the graphical presentation.

$f_{A}$ (factor for scattering effect of air). The effect of the air in scattering particles into the counter was determined by Zumwalt $(4)$ indirectly by the use of thin polystyrene films placed between the source and counter. The magnitude of the effect increases with distance from the counter and with decreasing energy of the beta particle. With the sample on the first shelf position $\left(0.5-\mathrm{cm}\right.$ air path), he found the factor to vary from 1.001 for $\mathrm{UX}_{2}$ $(2.3-\mathrm{Mev} \beta)$ to 1.005 for $\mathrm{Co}^{60}(0.3-\mathrm{Mev} \beta)$, and on the fourth shelf $(5.3-\mathrm{cm}$ air path), from 1.012 for $\mathrm{UX}_{2}$ to 1.060 for $\mathrm{Co}^{60}$.

$\mathrm{f}_{\mathrm{C}}$ (factor for effect of sample covering). In many cases samples are covered with thin protective plastic films. Since these are close to the sample, scattering effects are important, and the counting rate is usually increased somewhat over what would be observed if only absorption were taking place. This "forescattering" effect was first observed and qualitatively explained by Kovarik。(2) (Also see references $4,20$. ) The effect is a function of the thickness and atomic number of the absorbing foil, the energy of the beta radiation, and the distance between the sample and the detector. For a given covering material ( $3 \mathrm{mg} / \mathrm{cm}^{2}$ of polystyrene), Pappas $(20)$ found the forescattering factor to be negligible on the first shelf and equal for second, third, and fourth shelf positions. He defines the factor as the ratio of counts with the cover on the sample to that with the cover near the counter window (where it acts only as an absorber). The factor decreases with increasing energy of the beta particles from about 1.11 at $0.2 \mathrm{Mev}$ to 1.01 at $2.2 \mathrm{Mev}$. Zumwalt $(4)$ examined the forescattering effect (defined as the difference between the counting rate with absorber directly on the source and near the window expressed as a percentage of the counting rate of the sample with no absorber) for second and fourth shelf positions with various beta rays and absorbers. In general, the forescattering effect increases to a maximum with increasing thickness of absorber and then decreases again. The maximum tends to shift toward thicker absorbers as the beta energy increases and as the atomic number of the absorber decreases. The initial slope of the forescattering curves increases with decreasing beta energy and with increasing atomic number of the absorber. The maximum forescattering increases somewhat with decreasing geometry。

Studies of the angular distribution of scattered radiation(21) indicate that the spatial distribution follows a cosine law. The isotropic emission expected from an isolated source is modified, so that the radiation is con centrated in the direction of the detector.

Corrections for the effect of any covering on the sample may be made, for the first shelf position, by considering the cover as added absorber 
and extrapolating the absorption curve as in the case of the air and counter window correction (see $f$ W). At lower geometries, the scattering effects must also be taken into account. These act to compensate the loss by absorption in the cover and, in many cases, the two effects nearly cancel, giving a value of $f_{C}$ near unity.

$I_{B}$ (factor for effect of backscattering). Many experimental studies of the backscattering of electrons have been reported. $(2,4,5,22-32)$. These data indicate the dependence of the effect on the nature and thickness of the scattering material, the electron energy, and the geometry of the source.

The counting rate of a sample is increased by contributions from radiation initially directed away from the counter, but scattered back by the material on which the source is mounted. This contribution increases with the thickness of the backing to a saturation value which occurs at a thickness equivalent to about $15-25 \%$ of the range of the beta particles in the material (Figure 3 ).

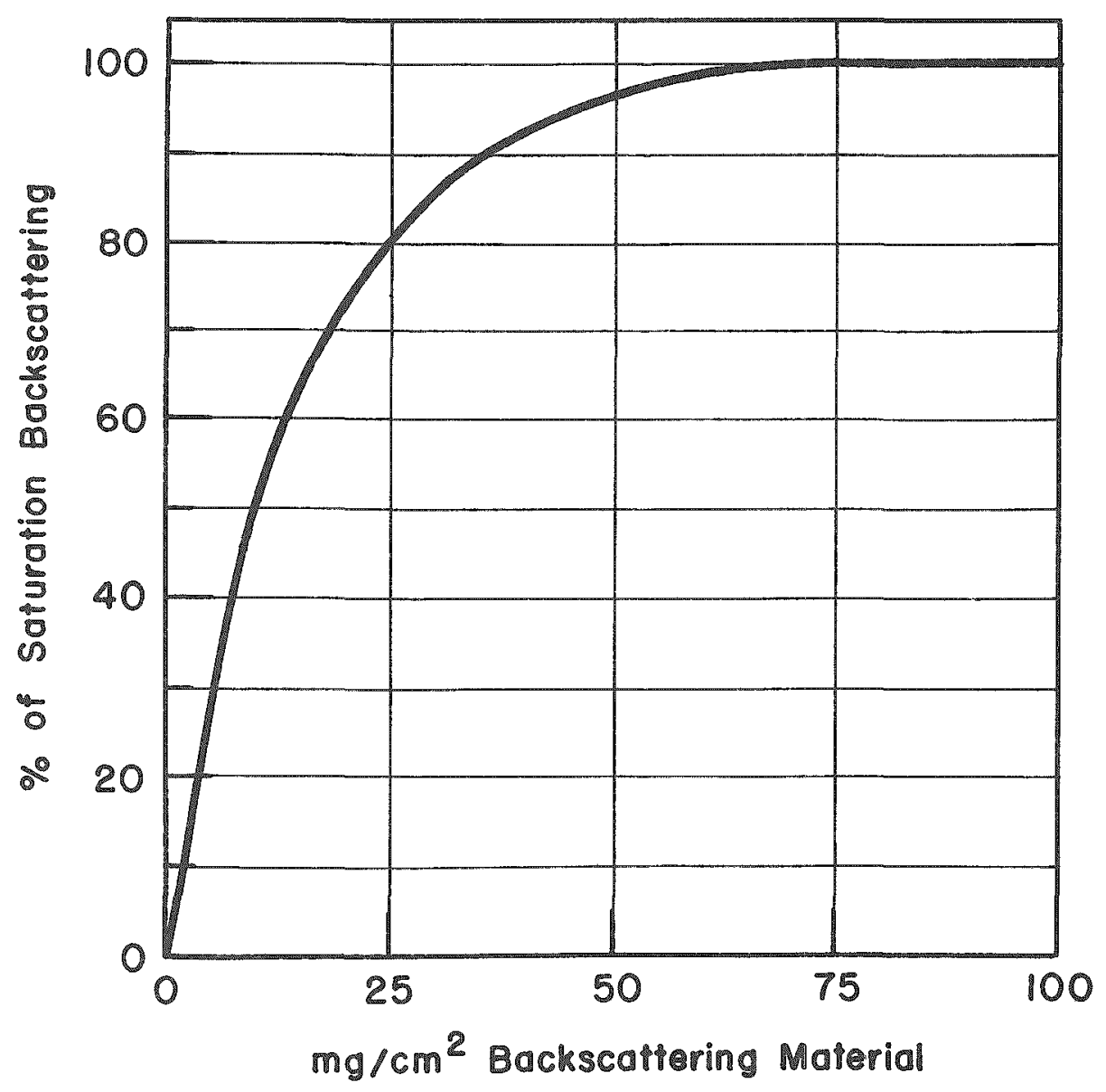

Figure 3

Typical backscattering "Growth" curve. 
(The fraction of the range at which saturation backscattering occurs decreases with increasing beta energy and, to a lesser extent, with increasing atomic number of the scatterer.) The per cent increase in counting rate at saturation increases with increasing atomic number of the scatterer (Fig ure 4). When appropriate corrections are made for the difference in absorption of the backscattered radiation (which is degraded in energy) in the air and counter window, it is seen that the saturation backscattering for a given scatterer is independent of energy over the range investigated $10.17 \%$ $1.7 \mathrm{Mev}) .(4,5,26)$

Most data on backscattering have been obtained with sources on thin films and scattering materials placed very close to or in contact with the film. Significant increases in the backscattering effect are obtained with the sources placed directly on the scattering materials, possibly as a result of surface imperfections.

The degradation in energy which takes place as a result of inelastic collisions in the process of backscattering is a function of the atomic number of the scatterer. $(4,17,26,30)$ Experimental data on the energy degradation of backscattered radiation indicate somewhat greater energy degradation on scattering from materials of low atomic number than from high. These data are presented in Figure 5 in terms of the relative half-thicknesses of scattered and unscattered radiation.

Experimental investigations of the angulax distribution of backscattered radiation have shown it to be anisotropic。 $(4,21,25,27,32)$ The angular anistropy is a function of the atomic number of the backscatterer, $(25,27,32)$ and Seliger (27) has shown that the distribution can be conveniently inter preted on the basis of "diffusion" and "side-scattering" effects, the former increasing with atomic number $Z$ while the latter is relatively constant. For material of low $\underline{Z}$ the energy degradation is much larger at a scattering angle of $90^{\circ}$ than at $10^{\circ}$, whereas for high $\underline{Z}$ both are about the same.

$\mathrm{f}_{\mathrm{H}}$ (factor for the effect of the housing). Scattering from the walls of the counter shielding and sample support structure contributes negligibly to the observed counting rate of a sample mounted on thin backing except for positions neax the base of the housing. (4) The effect increases with energy of the beta ray, and on the fourth shelf $(11 \mathrm{~mm}$ from the base of an aluminum lined, lead shield and about $53 \mathrm{~mm}$ from the tube window), Zumwalt observed an increase from a factor of 1.045 for $\mathrm{CO}^{60}$ to 1.102 for UX 2 .

This effect is dependent on the materials used for construction of shield linings and sample supports. Materials of low $\underline{Z}$ should be employed wherever possible to minimize the scattering. 


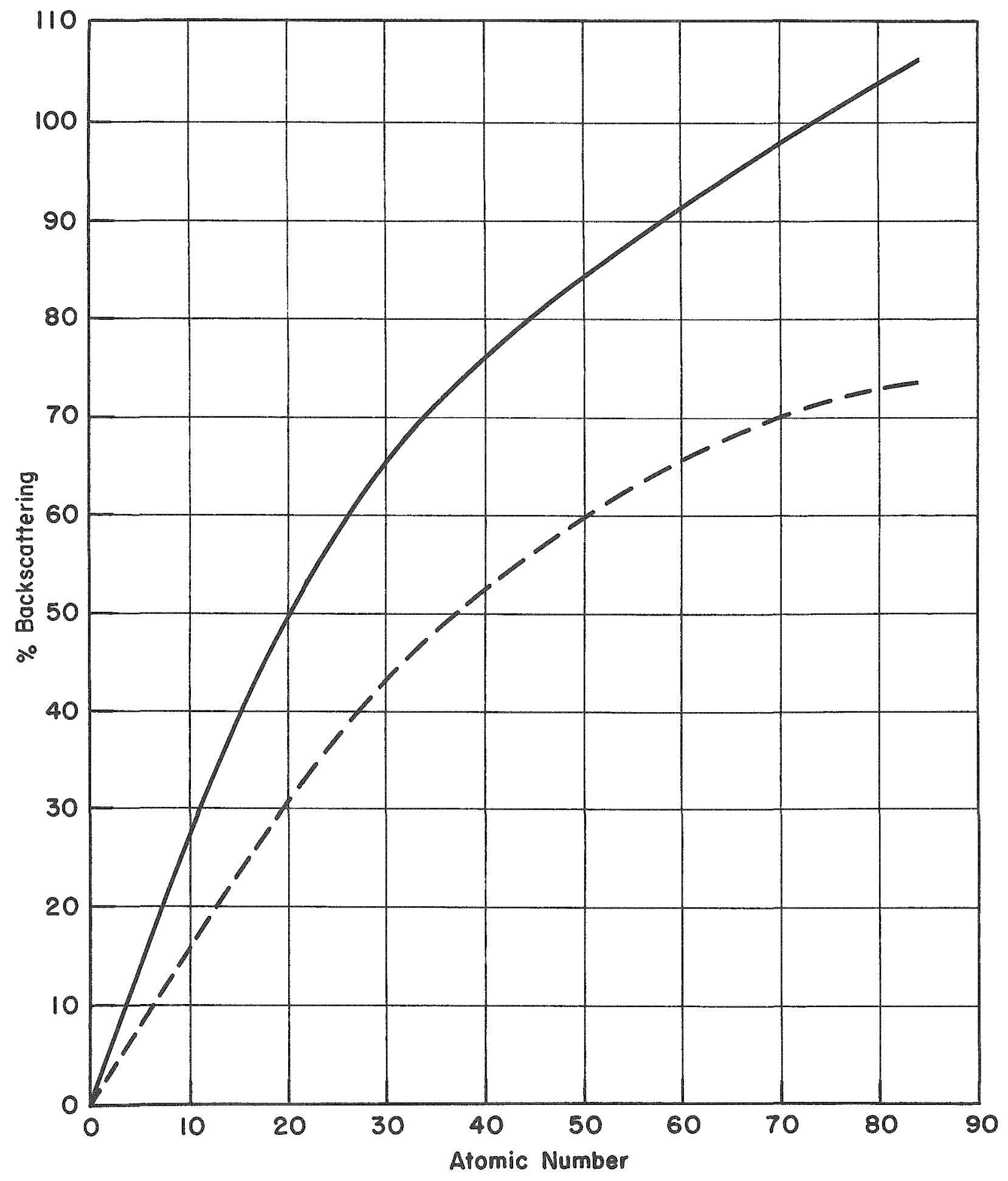

Figure 4

Saturation backscattering of $\beta$ radiation as a function of the atomic number of the backscattering material. The solid line represents data for the source directly on the metal surface and the broken line represents data for the source on a thin film separating it from the metal. 


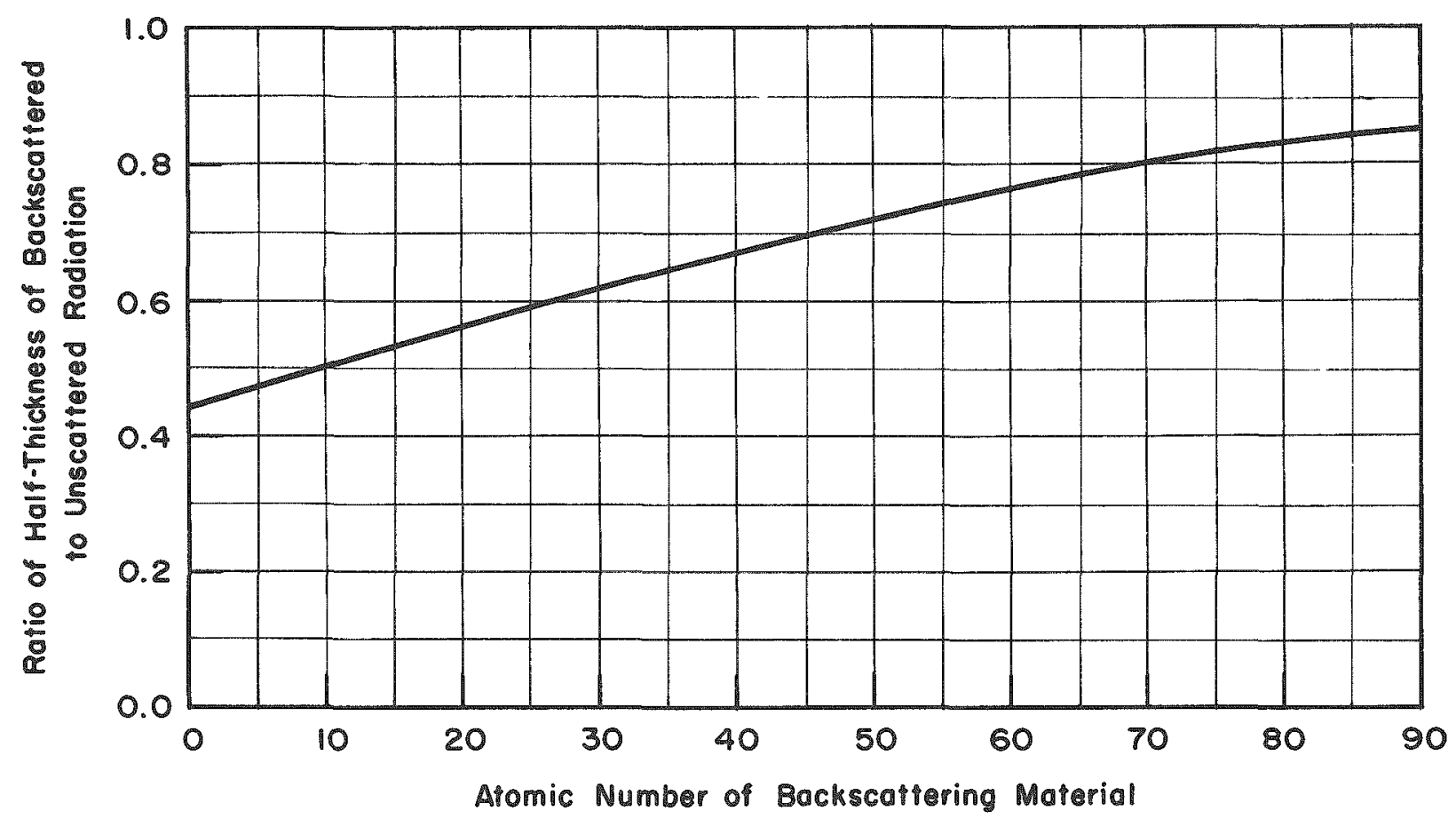

Figure 5

Energy degradation of backscattered radiation as a function of the atomic number of the backscattering material.

$\mathrm{f}_{\mathrm{S}}$ (self-absorption and self-scattering factor). It is not always convenient or possible to prepare samples which are effectively "weightless." In many experiments, samples of low specific activity are encountered or carriers are used, and it is necessary to count samples of appreciable weight. In such samples scattering and absorption of radiation takes place within the source material itself. The effects depend in a complex manner on sample thickness, the effective atomic number of the source material, the energy of the radiation, and the geometry. The largest error in the determination of beta disintegration rates probably lies in the determination of $f_{S}$.

Although a number of attempts have been made to generalize the effects of self-absorption and self-scattering and some equations and graphs have been published, $(25,33-55,110)$ these are, in general, applicable only to experimental conditions duplicating those under which the effects were studied. The published data are, of course, extremely useful in illustrating the nature of the effects and they can be used for approximate corrections. For more accurate work, however, the corrections should be determined empirically by each investigator.

Self-absorption in thick samples is fairly well represented by an equation which assumes exponential absorption and no self-scattering. The 
activity, $A_{t}$, observed from a sample of thickness, $t \mathrm{mg} / \mathrm{cm}^{2}$, is given by $(39,40)$

$$
A_{t}=A_{O} \frac{\left(1-e^{-\mu t}\right)}{\mu t},
$$

where $A_{O}$ is the activity which would be observed from a weightless source, and $\mu$ is the mass absorption coefficient measured in $\mathrm{cm}^{2} / \mathrm{mg}$. For geometries of less than $2 \pi$, the angular distribution of scattered radiation gives rise to the "forescattering effect" discussed above in connection with added absorbers. With increasing weight of a uniformly radioactive source, the specific activity (counts/minute/mg) rises initially as a result of selfscattering, reaches a maximum (at about 5-10\% of the range of the $f$ radiation), and then decreases in accordance with the self-absorption equation (Figure 6). It is obvious that if sufficient data are not obtained at small thicknesses, or if the simple self-absorption equation is applied to data beyond the maximum, the extrapolated counting rate at zero weight may be in error. In an extreme case the exror may be as much as a factor of two.

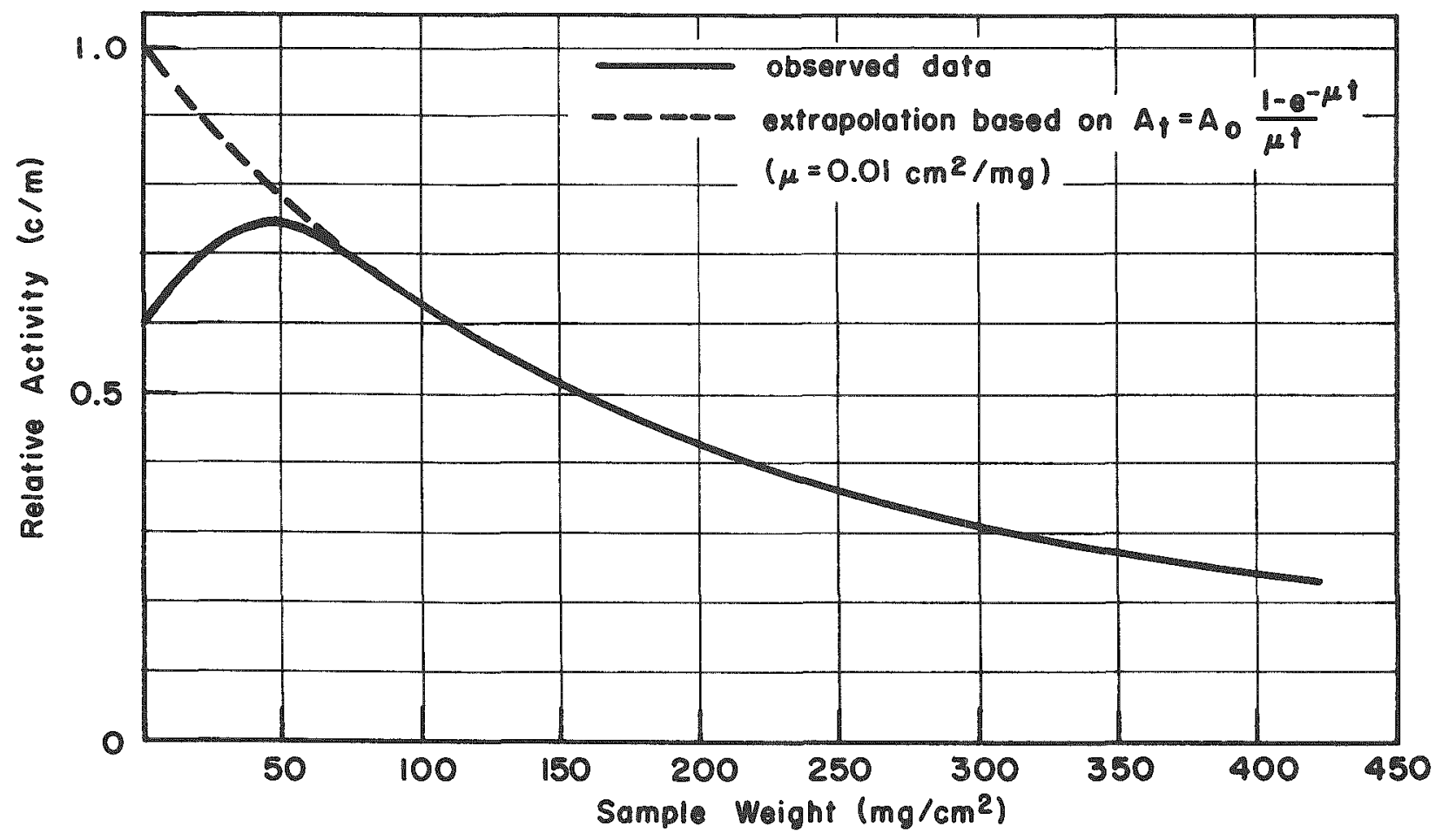

Figure 6

Illustration of self-absorption and self-scattering effects as a function of sample weight. 
Baker and Katz(52) have shown that such a curve can be described as the product of self-scattering and self-absorption curves. The self-scattering curve is determined from measurements of the angular distribution of the radiation from thick sources, and is expressed in terms of the ratio, $\frac{N_{O}, x}{\bar{N}_{X}}$, where $N_{O}, x$ is the activity measured in a direction normal to the plane of the sample of thickness $x$, and $\bar{N}_{X}$ is the activity averaged over all angles. Although sufficient data to establish a dependence on atomic number were not obtained, a single curve described $\frac{N_{O}, x}{\bar{N}_{X}}$ as a function of sample thickness (expressed as fraction of the range). Walton $(53)$ derived equations describing the combined effects of self-absorption, self-scattering and back-scattering, which account fairly well for observed results. It is significant to note that sources with smooth flat surfaces were essential in order to obtain reproducible results in Walton's work. This illustrates the necessity of maintaining standardized conditions in relative counting rate determinations. A similar study has been carried out by Lerch. (54)

The foregoing discussion of the factors which may contribute to er rors in beta counting illustrates the complexity of the problem of determining the disintegration rate of even a simple beta emitter from a thick source. When other radiations from the nuclide of interest or genetically related species are present the contribution of these to the observed counting rate must be evaluated. In general, absorption techniques are combined with decay measurements to analyze multi-component systems, and the radiations of interest are corrected for the effect involved. These measurements may introduce additional errors arising from uncertainties in the half-lives of the activities involved and difficulties in analyzing absorption curves (Figure 7).

Suggested Techniques for End Window Counting

Absolute Beta Counting. The most accurate method of determining beta disintegration rates using end window counters is to prepare essentially weightless sources mounted on thin backing and to count in a position of known or calibrated geometry. If the geometry has been determined by the use of some standard (such as RaE(6)) or is calculated, it will be necessary to apply corrections for absorption and scattering effects. Of course, if a geometric efficiency for the radiation has been determined, no corrections are needed (other than background and any resolution loss). Such techniques should give errors of the order of $5 \%$ or less.

When it is necessary to determine absolute disintegration rates from thick sources, within a reasonable degree of error, e.g., the order of $10 \%$ or less, it is essential to prepare calibration standards. A high specific activity source of the activity of interest should first be prepared 


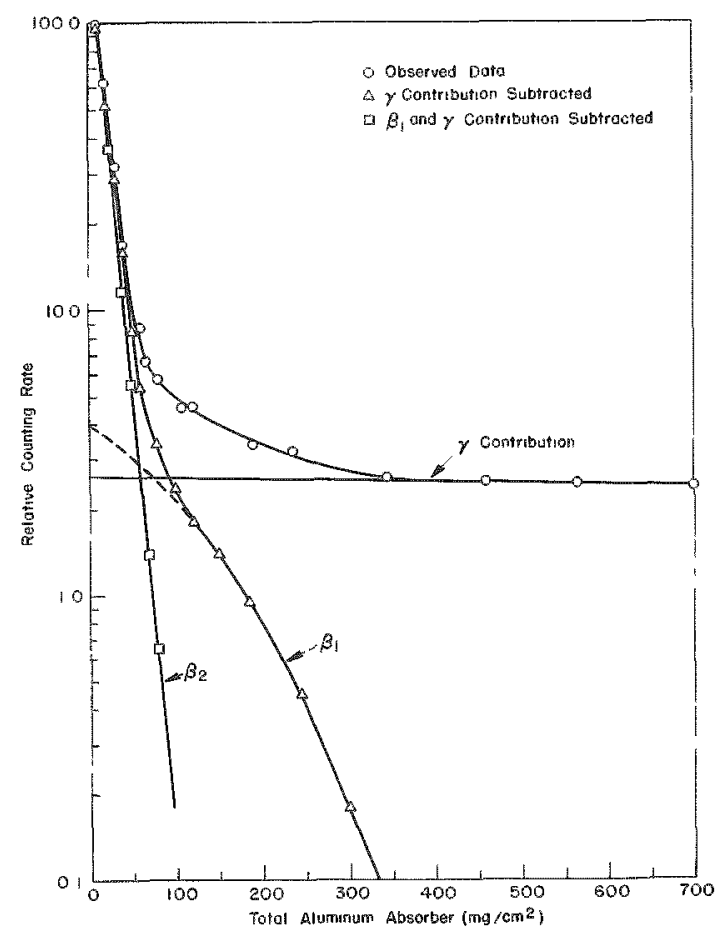

Figure 7

Analysis of a complex absorption curve.

and its disintegration rate determined by $4 \pi$ counting, coincidence counting if applicable), or the counting of a weightless source on thin backing in a position of known geometry. Equal aliquots of the activity are added to solutions containing varying known amounts of the element of interest. The compound to be measured is precipitated, the chemical yield determined, and the sample mounted and counted under the desired conditions. The observed counting rates are corrected for chemical yield, background, and resolution losses. A comparison of the observedactivity of these samples with the known disintegration rate of the aliquot added gives an overall counting efficiency for the conditions of the measurement. The counting efficiency for each sample maybe plotted graphically as a function of sample weight, and a calibration curve will the reby be obtained which can be readily applied to the determination of disintegration rates of other samples measured under the same conditions. It is, of course, important to ensure reproducibility of the sample mounting technique by determining a number of samples at each weight. Similar curves must be obtained for each different activity or compound to be measured.

If it is not possible to obtain a source of high specific activity, or if errors of the order of $20 \%$ will suffice, correction factors or equations reported in the literature $(25,31-55,110)$ may be used. In general, these 
have been determined under conditions which should not be difficult to reproduce rather closely. Examples of such correction factors for $\mathrm{S}^{35}$

$\left(\beta^{-}=0.167 \mathrm{Mev}\right.$, precipitated as $\left.\mathrm{BaSO}_{4}\right)$ and $\mathrm{P}^{32}\left(\beta^{-}=1.7 \mathrm{Mev}\right.$, precipitated as $\mathrm{MgNH}_{4} \mathrm{PO}_{4} \cdot 6 \mathrm{H}_{2} \mathrm{O}$ ) are given in Figure 8 . The curves represent data (103) for precipitates of different weights collected over a $2-\mathrm{cm}^{2}$ area on filter paper, mounted on cardboard cards, and covered with rubber hydrochloride of $0.5-\mathrm{mg} / \mathrm{cm}^{2}$ thickness. The samples were counted in the first shelf position (geometry factor $=36 \%$ of $4 \pi$ ) of a standard end-window flow proportional counter assembly using a counting tube with a $0.6-\mathrm{mg} / \mathrm{cm}^{2}$ window. The disintegration rate of an aliquot of a solution of the radionuclide was determined by $4 \pi$ counting. Equal aliquots were incorporated in precipitates of varying weight and the counting rates determined.

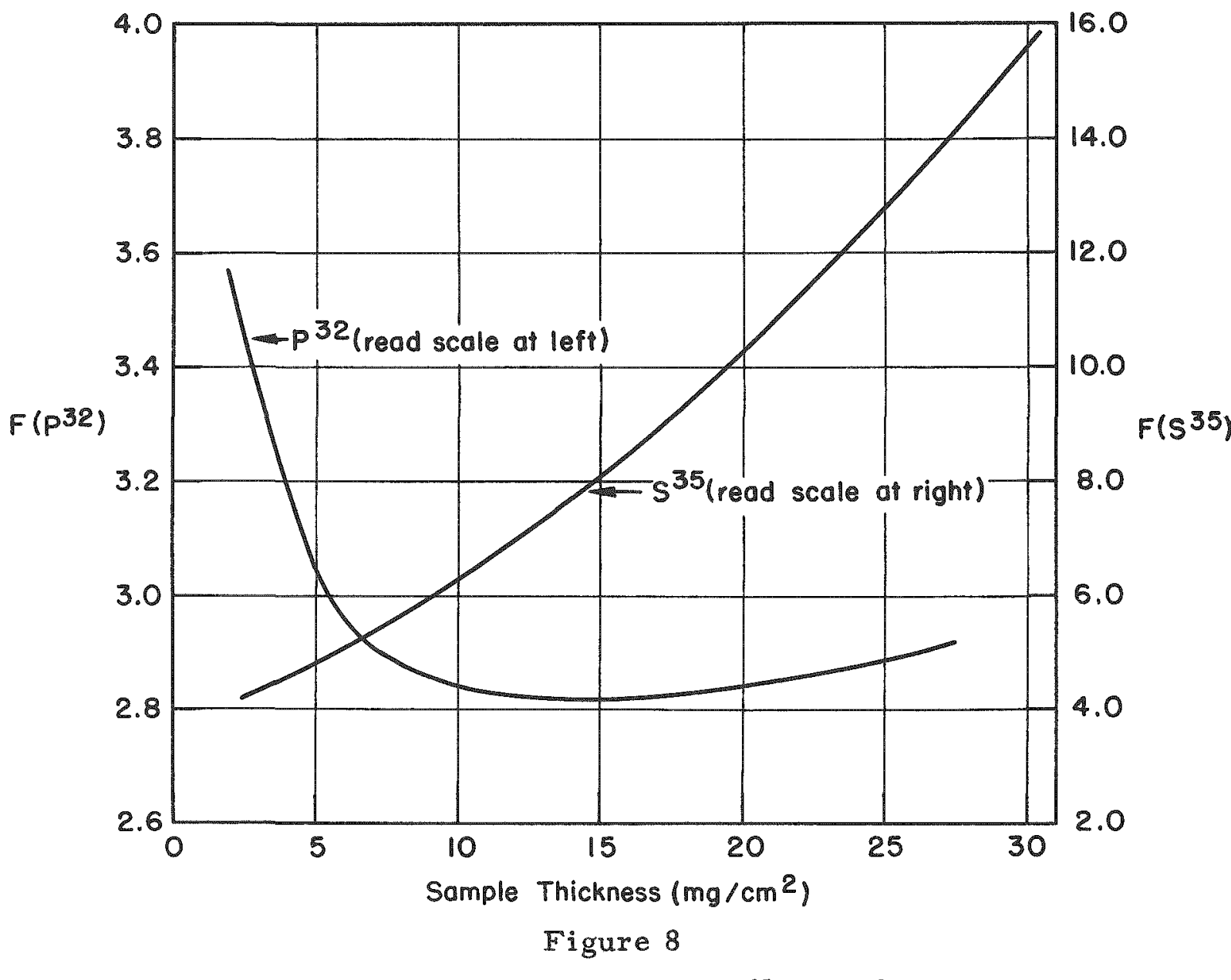

Empirical correction factors for $\mathrm{S}^{35}$ and $\mathrm{P}^{32}$ to obtain disintegration rates from observed counting rates. 
The observed counting rates were corrected for the chemical yield in the precipitation (i.e., the fraction of the added carrier actually mounted) and for the absorption effects of the sample covering, the air path, and the counter window. The correction factor, F, represents the ratio of the disintegration rate to the corrected counting rate of the samples. Figure 9 gives the correction factor, $F$, as a function of $\beta$-particle energy for precipitates of $10 \mathrm{mg} / \mathrm{cm}^{2}$ thickness. Differences in the average effective atomic number of precipitates other than those used in determining the curve of Figure 8 may be ignored for approximate calculations. The factor is essentially constant for source thicknesses over the range $5-15 \mathrm{mg} / \mathrm{cm}^{2}$.

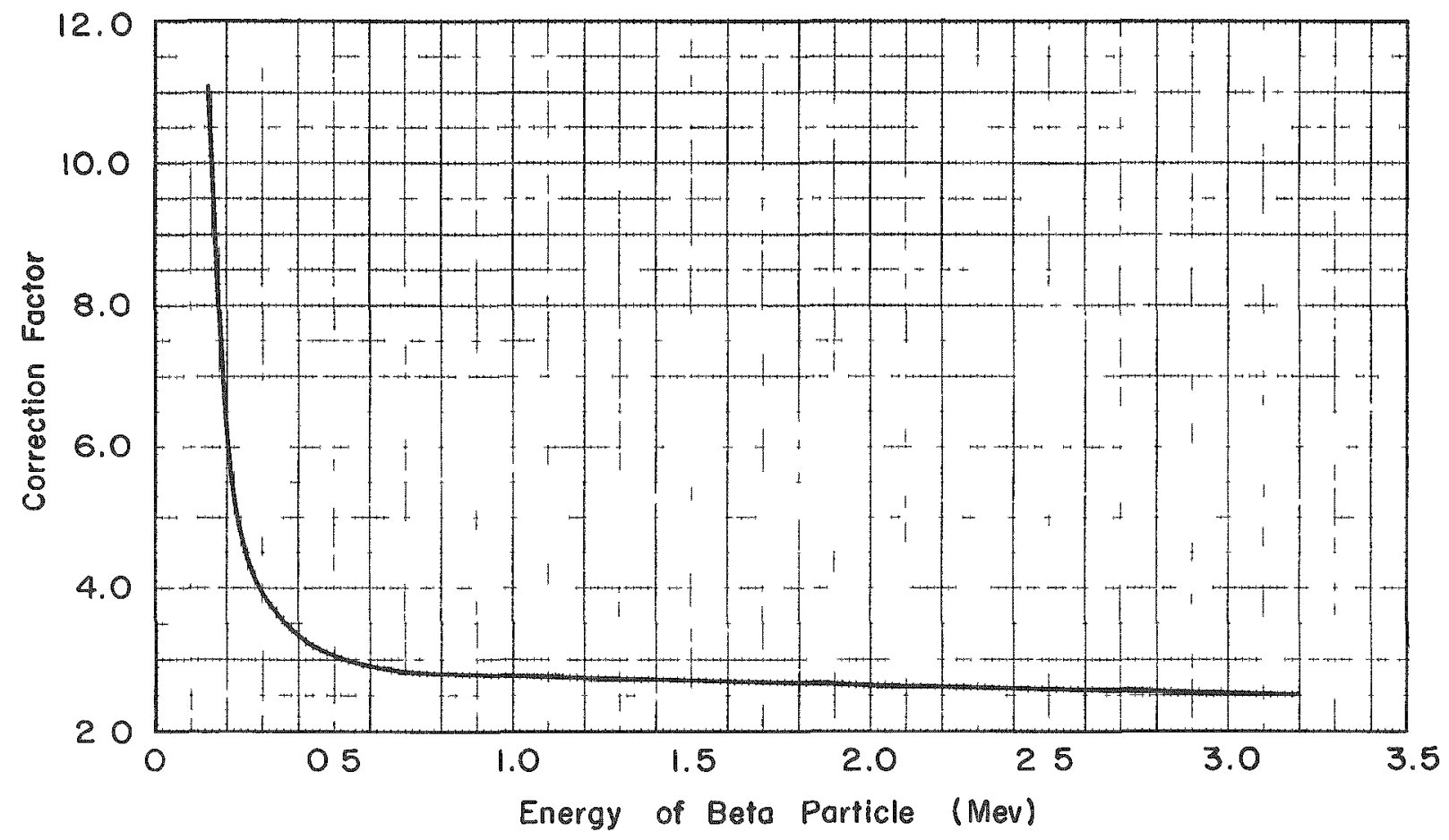

Figure 9

Empirical self-absorption, self-scattering correction factor as a function of beta energy for $10-\mathrm{mg} / \mathrm{cm}^{2}$ sources.

In some instances if the activity level permits, a solution of a thick sample may be dissolved and relatively thin sources prepared from small aliquots.

Relative Counting. The most important consideration in relative counting is the reproducibility of sample preparation and mounting techniques. In particular, sample areas and thickness should be the same. 
Reproducibilities with about $2 \%$ error may be expected for the relative count ing rates of properly prepared samples. A number of techniques for preparing reproducible samples have been reported. $(38,44,45,47,48,51,53,104,105,110)$ In comparing the counting rates of samples which may differ in chemical composition, weight, size, mounting, etc., corrections must be appliedas outlined above, and, consequently, the precision of the result may be somewhat less. In general, it is desirable to prepare either very thin samples, in which the corrections are minimized, or samples thick enough to be approaching saturation of the effects, since these can be most readily made reproducibly.

$2 \pi$ Counting

Counting under conditions of a solid angle of $2 \pi$ radians may be carried out with external or internal samples. Since the effects of the anistropic distribution of scattered radiation are minimized at such high geometry, the major effects of concern are true self-absorption and back-scattering (including that from the sample itself). These are treated in the same manner as described previously for end-window counting - namely, by minimizing the effects if possible, or by establishing empirical correction factors. $(55-57,109)$

$2 \pi$ counting is particularly advantageous for samples of low activity, and a number of instruments have been utilized for this purpose. Samples may be mounted externally by wrapping around glass - walled cylindricaltype Geiger counters, (118) or internally in a flow-type proportional counter or screen-wall counter. (58) The internal counters are, of course, desirable and sometimes necessary for the counting of very low energy betas. These instxuments may be calibrated as described in the previous section to determine a counting yield (or efficiency).

A typical efficiency curve(103) for a flow-type internal proportional counter using weightless samples mounted on 5 -mil Pt foils is shown in Figure 10. The fact that the efficiency falls below $50 \%$ at very low energies is probably the result of self-absorption in the sources. It is extremelydificult to prepare sources which are essentially "weightless" for radiation of such low energy. Somewhat different results would be obtained with different mounts as a result of backscattering differences. Backscattering corrections have been determined $(106-108)$ for internal $(2 \pi)$ flow proportional counters as a function of the atomic number of the mounting plate, and such a curve(106) is given in Figure 11 , where the backscattering factor $F_{B}$ represents the ratio of the observed counting rate of a known aliquot of a solution of the radionuclide to one-half of the known disintegration rate of the aliquot (determined by $4 \pi$ counting). In many designs of $2 \pi$ counters, materials of construction are close to or in contact with the sample mount. Thus, it may not be possible to eliminate or minimize backscattering by the use of 
- .

s.

25

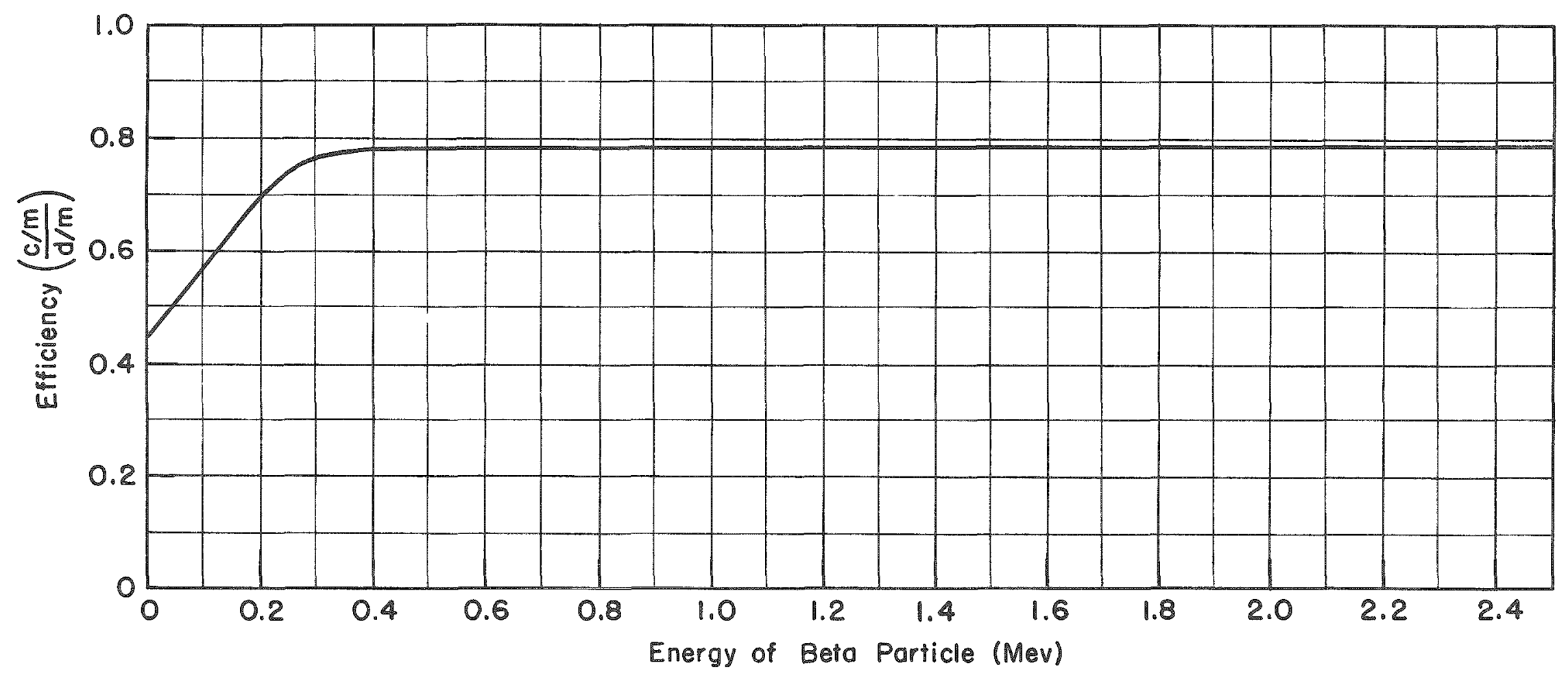

Figure 10

Counting efficiency of an internal ( $2 \pi$ ) flow proportional counter for samples mounted on $5 \mathrm{mil} P t$. 


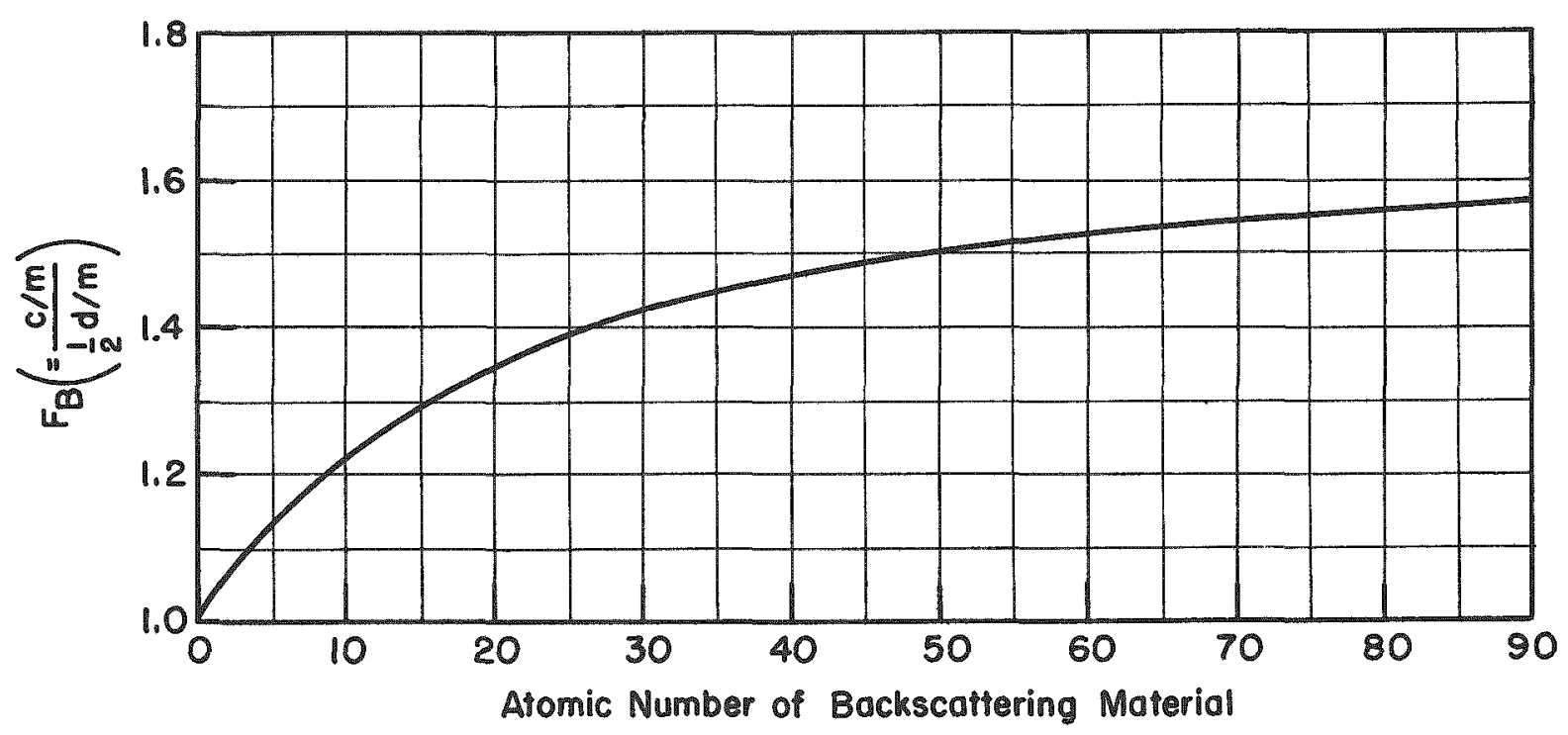

Eigure 11

Backscattering at $2 \pi$ Geometry.

thin films for mounting samples, and mounting plates thick enough to give saturation backscattering should be used. Even with the same mount material some differences in backscattering may be observed due to differences in surface conditions, and where high precision is desired this should be borne in mind.

Absolute beta disintegration rates may be determined using internal (2T) counting technique, with suitable calibration, to an accuracyinvolving errors of the order of a few per cent.

Suttle and Libby $(56)$ have shown that under conditions of close cylindrical geometry, such as obtains in the use of a screen-wall counter, the assumption of purely exponential absorption is valid for thick sources of simple beta emitters. For a sample thick with respect to $\lambda$, the reciprocal of the mass absorption coefficient of the radiation, the absolute specific radioactivity in disintegrations per minute per gram of sample is given by

$$
\sigma=\mathbb{R G} / \mathrm{A} \lambda,
$$

where $R$ is the observed counting rate in counts per minute, $A$ is the area of the sample in square centimeters, $\lambda$ is the reciprocal of the absorption coefficient of the radiation in units of grams per square centimeters, and $G$ is the ratio of $4 \pi$ to the solid angle subtended by the sensitive counter 
volume at the source. (It is as sumed that $G$ is not seriously dependent on the depth of sample and has been averaged over its area.) With this technique absolute disintegration rates may be obtained with an error of about $5 \%$.

$4 \pi$ Counting

If one event were recorded for each disintegration of a radioactive nuclide, a direct measure of the absolute disintegration rate would be readily obtained. In practice, the $4 \pi$ counter comes closest to achieving this ideal condition. A number of designs of $4 \pi$ counters have been described(59) utilizing twin, facing Geiger ox proportional counters with the sample mounted between them, and multiple counter arrangements. (52) Methods based on charge collection have also been reported. $(60)$ The sample may also be homogeneously distributed through the detector in the gas phase or in solution (see sections on Internal Gas Counting and Beta Scintillation Counting).

When the two halves of a $4 \pi$ counter are connected in parallel, coincident events (such as betas, conversion electrons, secondary (delta) rays, $X$ rays or gammas) will be recorded as a single pulse. The detec tion efficiency of Geiger and proportional counters for particles is very close to $100 \%$, so that if particle emission (which may be $\beta^{-}, \beta^{+}$, conver sion or Augex electrons) accompanies every disintegration (or a known fraction of the disintegrations) the $4 \pi$ counter, in principle, will record directly the disintegration rate regardless of the complexity of the decay scheme. If excited states occur with lifetimes longer than the resolving time of the instrument, these will, of course, be recorded as independent events and their contribution to the observed count must be determined from a knowledge of the decay scheme. Likewise, the presence of isotopic or genetically, related radioactivities of the nuclide of interest will con tribute to the observed counting rate, and their contribution must be evaluated. As is necessary in all counting methods, background activity and resolution losses must be corrected for.

Although the $4 \pi$ counter offers a versatile and convenient method of determining absolute disintegration rates, a few sources of error must be considered - namely, absorption of radiation in the mounting film which separates the two counters, the possibility of a counting efficiency of less than $100 \%$, and self-absorption in the sample.

Pate and Yaffe(59) have carried out a systematic survey of $4 \pi$ counting techniques using twin, facing proportional counters. They have examined the problem of source-mount absorption correction and have pointed out errors in previous publications in connection with this correction. Three techniques have been used to correct for this effect: (1) a sandwich" procedure $(61)$ in which the counting rate is determined for the 
sample on a mount of known thickness and another determination made with a film identical to the mount covering the sample: (2) a calculation using simplified assumptions of scattering and absorption effects and measurements of $2 \pi$ and $4 \pi$-single and sandwiched film counting rates:(62) and (3) an absorption curve method(63) in which the source is mounted on a thin film (5 to $10 \mu \mathrm{g} / \mathrm{cm}^{2}$ ) and the counting rate observed as a function of increasing mounting film thickness. Increased thicknesses of film are obtained by allowing thin films of known thickness to adhere to the back of the mounting film. The "absorption curve" obtained may be extrapolated to zero thickness to obtain the disintegration rate.

For beta energies about a few hundred kev, any of these methods work quite well since the corrections are small. However, at lower energies the absorption curve technique appears to be the most accurate. Pate and Yaffe estimate that disintegration rates can be determined to an accuracy of about $\pm 0.2 \%$ for energies as low as $67 \mathrm{kev}$ and probably to higher accuracy at somewhat higher energies. Their data are reproduced in. Figure 12 in which the ratio of observed counting rate to disintegration rate $\left(\mathrm{N}_{\mathrm{T}} / \mathrm{N}_{\mathrm{O}}\right)$ is given as a function of beta end-point energy for a series of film thickness. These data may be used directly to abtain the source mount correction for beta rays of known energy. It should be remembered that beta-ray spectra may differ in shape even for identical end-point energies, and unless the correction is to be applied to a nuclide for which data is given in Figure 12, the accuracy of the disintegration rate value will not be as high as if an empirical determination of the absorption curve is made.

Geiger-Muellex and proportional counters have sensitive volumes which are very nearly $100 \%$ efficient, but, as discussed previously, there may be regions of low field strength and a consequent loss in detection efficiency. This is particularly true in internal counters when the source is mounted on a nonconducting film on which charge may accumulate and cause field distortion. Thin, evaporated metal coatings should be used on all mounting films.(59b)

The most difficult correction to make is that for self-absorption in the source. The preparation of very thin sources has plagued many fields of investigation, particularly when known aliquots must be deposited. Examinations with optical and electron microscopes show evaporated sources to consist of small clusters of crystals. Additional solid (as carrier) might fill in the spaces between clusters, giving a more uniform, but not necessarily thicker source. Thus, the technique of determining a self-absorption curve by depositing equal aliquots containing increasing weights of carrier may not be applicable. Optical measurements of $\mathrm{S}^{35}$ and $\mathrm{Co}^{60}$ sources (59e) indicate that with increasing weight of carrier the number and size of crys tals increase. The problem of source preparation is not easily solved, and. at present, the best that can be done, if a self-absorption curve does not appear applicable, is to examine sources of low energy betas and estimate 


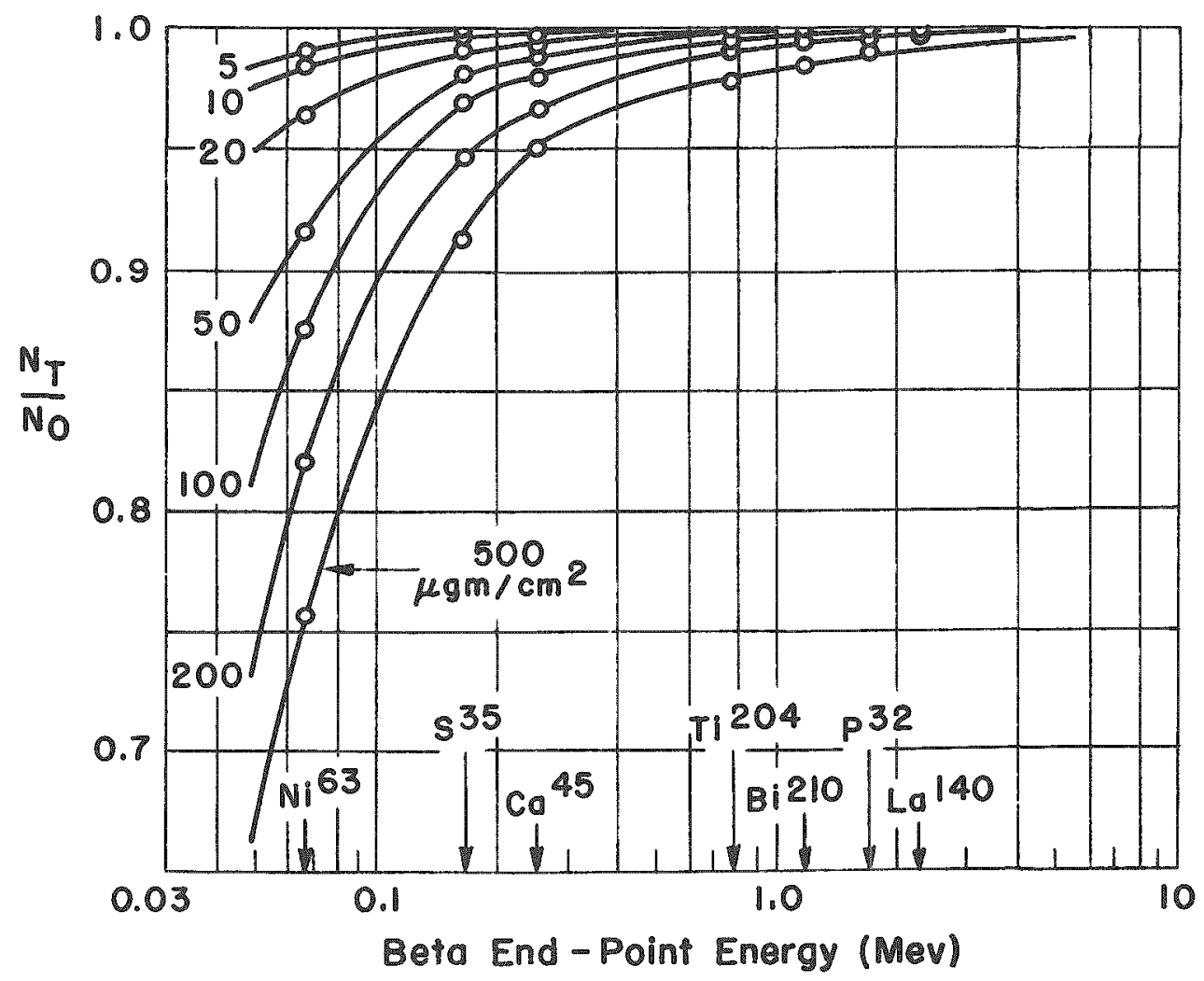

Figure 12

Absorption parameter $\left(\mathbb{N}_{T} / N_{O}\right)$ as a function of beta end-point energy, for a series of film thicknesses.

the self-absorption loss from the density of the deposit. Corrections of the order of a few per cent may be expected, e.g., for $\mathrm{Co}^{60}(0.3 \mathrm{Mev})$. They may, of course, be less for more energetic and greater for less energetic radiation.

Methods for preparation of thin films and samples are given by Slatis. (119) The most satisfactory method for preparation of thin uniform samples appears to be by volatilization from a hot filament. $(59 \mathrm{e}, 64)$

The $4 \pi$ counting technique is the most generally applicable method for the determination of disintegration rates of radioactive sources and is to be recommended for the most precise work. Methods of measurement 
for the calibration of a number of radioactive sources prepared at the National Bureau of Standards are reviewed by Seliger and Schwebel. (65) This report illustrates the application of the $4 \pi$, coincidence, and internal gas-counting techniques (see below).

Internal Gas Counting

Another type of $4 \pi$ counting may be achieved by incorporating the activity in the gas phase with the counting mixture in a Geiger or proportional counter, (66-68) or by the use of ionization chambers. (69) For the determination of absolute disintegration rates, cylindrical tubes are gen erally used and filled with measured quantities of counting and radioactive gases. Each measurement requires a new filling, and the counter characteristics must be checked to ensure proper operating conditions for each determination.

In addition to the corrections for background and resolution losses, the effects of field inhomogeneity at the insulated ends of the sensitive volume (end effect) and the escape of radiation originating near the cathode wall before causing ionization (wall effect) must be considered. End effect corrections for brass wall counters having flat Lucite ends have been investigated as a function of the length to diameter ratio of the counter. (70)

The end effect is dependent on the tube construction and may be determined from measurements in two counters of identical construction and diameter but of different length. The wall loss is a function of the energy of the radiation and is inversely proportional to the pressure in the counter and to the diameter of the tube. Backscattering and secondary electron emission from the wall tend to compensate the wall loss. The end and wall effects may be represented as additive correction terms on the actual bare wire length (L) and tube diameter (D). The active volume of the counter, V, is then given by

$$
V=\frac{\pi(D+d)^{2}(L+\ell)}{4}
$$

where $\mathrm{d}$ and $\underline{\ell}$ are the corrections to the diameter and wire length, respectively. These corrections may be determined by measurements with coun ters of constant length and varying diameters and vice versa or by measurements with standardized gas samples.

By employing a movable and a fixed glass shield around the central wire anode, Eidinoff(71) has shown that the end effect may be eliminated.

When proper care is exercised in internal gas counting, absolute disintegration rates may be determined with errors of the order of $1 \%$ and reproducibilities to within $0.5 \%$. 
External Gas Counting

Gas samples may be prepared in cells which can be counted externally in the same manner as a solid sample. $(72,73)$ Corrections for absorption and scattering effects as well as geometry must be made to determine disintegration rates. It is recommended that such cells be calibrated with gases of known disintegration rate if they are to be used for absolute measurements. For relative measurements, external gas counting may prove more convenient than internal counting, and it is only necessary to maintain reproducible conditions to obtain results of high precision.

Coincidence Counting

The coincidence counting technique $(9,74-76,120)$ may be used for absolute disintegration rate determinations in those cases where beta and gamma rays are emitted in sequence within the resolving time of the apparatus (usually of the order of $10^{-6}$ second). Although the method may, in principle, be applied to any known decay scheme, it is generally limited to simple systems of a single beta particle followed by one or more gamma rays. The convenience and accuracy of the method rapidly decreases with increasing complexity of the decay scheme.

If a point source of activity (which decays through a single beta followed by a single unconverted gamma) is placed between a beta counter and a gamma counter (shielded so as to be insensitive to beta particles) and the counters are connected through a coincidence circuit, data sufficient for a determination of the absolute disintegration rate may be obtained. A schematic diagram of the apparatus is given in Eigure 13. The counting rate in the beta counter, $\mathbb{N}_{\beta}$, is given by

$$
N_{\beta}=N \in \beta
$$

where $\mathrm{N}$ is the disintegration rate and $\epsilon_{\beta}$ is the over all efficiency of the counter for the beta particles (including geometry). Likewise,

$$
N_{\gamma}=N \epsilon_{\gamma}
$$

where $\mathbb{N}_{\gamma}$ is the gamma counting rate and $\epsilon_{\gamma}$ is the efficiency of gamma counting. Since the coincidence counter registers only when a beta and gamma count occur simuitaneously in the two counters, the counting rate of true coincidences, $N_{c}$, will be

$$
N_{c}=N \epsilon_{\beta} \in \gamma
$$

(assuming the coincidence circuitry has been adjusted to give unit coincidence efficiency). Then

$$
\mathbf{N}=\mathrm{N}_{\beta} \mathrm{N}_{\gamma} / \mathrm{N}_{\mathrm{C}}
$$




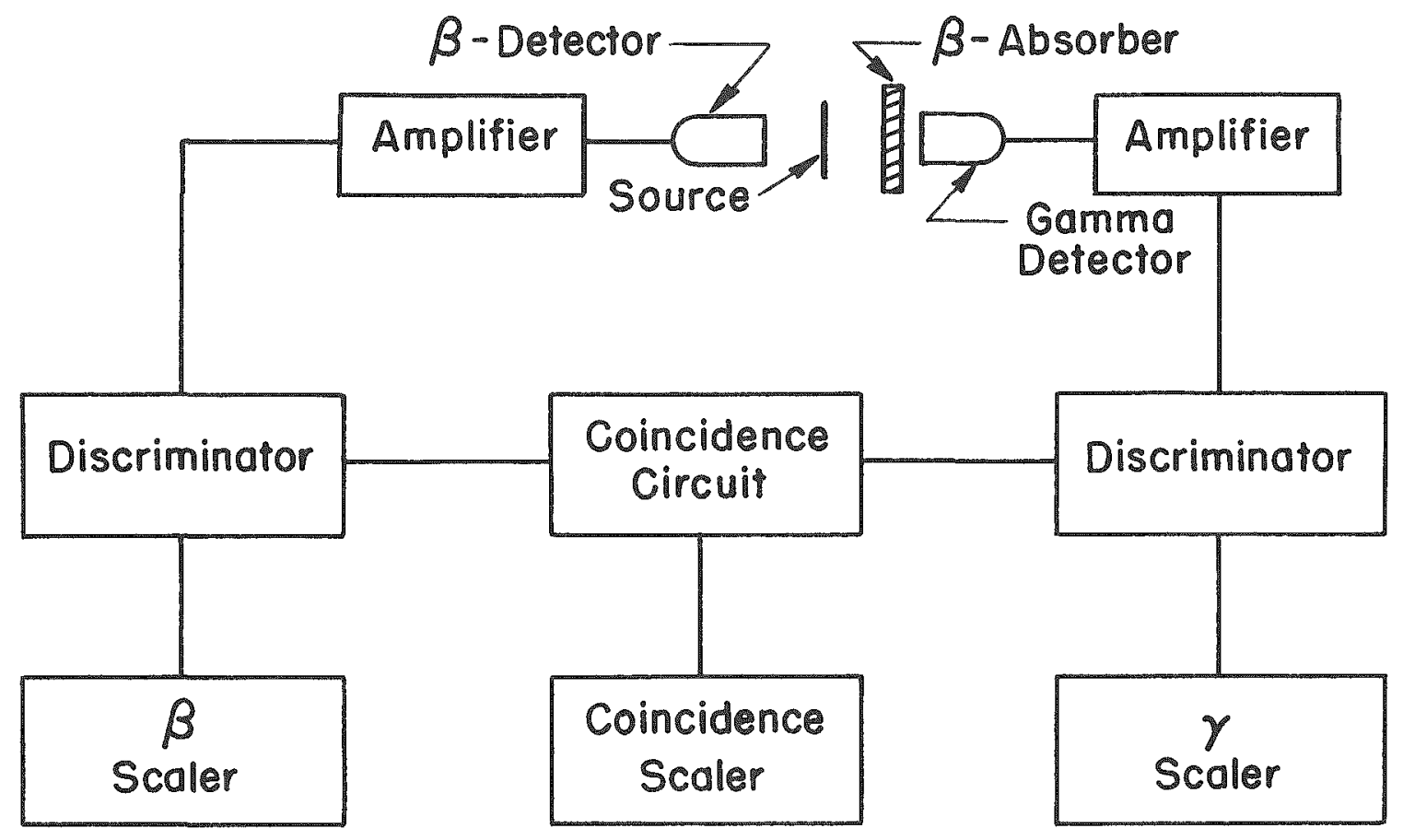

Figure 13

Schematic diagram of coincidence counting equipment.

$\mathrm{N}_{\gamma}, \mathrm{N}_{\gamma}$, and $\mathrm{N}_{\mathrm{c}}$ must be derived from the observed counting rates by the application of a number of corrections. These include corrections for chance (random) coincidences, backgrounds, presence of conversion electrons, any angular correlation, and dead time losses. In addition, for the coincidence method to be valid, at least one counter must have a constant efficiency (i.e., be equally sensitive) to radiations from all parts of the source. In general, the corrections are dependent on the particular detectors and electronic circuitry employed. It is advisable to use detectors which exhibit high efficiency for one radiation and low efficiency for the other. Thus the beta counter should be a Geiger, proportional, or thin crystal scintillation counter, all of which are highly efficient for betas, but have low gamma counting efficiency. A number of types of gamma counter having fairly high efficiencies have been developed. Among these, the gamma scintillation counter using thallium-activated $\mathrm{NaI}$ is perhaps the best. Beta detection in the gamma counter is prevented by the interposition of a suitable absorber.

Since the pulses arriving at the coincidence circuit have finite widths, two independent counts (not associated with the same disintegration) mayoverlap and give rise to a coincidence pulse. This random coincidence rate is given by

$$
\mathrm{C}_{\mathrm{R}}=2 \pi \mathrm{N}_{\beta} \mathrm{N}_{\gamma},
$$


where $2 T$ is the resolving time of the system and $N \beta$ and $N \gamma$ are the observed singles counting rates in the beta and gamma counters, respectively. [It should be noted that when the observed coincidence rate $N_{c}$ is not small with respect to the observed singles rates $N_{\beta}$ and $N_{\gamma}$, the above expression for $C_{R}$ must be modified to $\left.C_{R}=2 \tau\left(N_{\beta}-N_{c}\right)\left(N_{\gamma}-N_{c}\right).\right](77)$ The resolving time may be determined by the use of two independent sources suitably shielded from each other so that no scattered or coincident radiation is registered. Any coincidences recorded, then, will be primarily due to a background of cosmic ray showers and the finite resolving time of the circuit. Sources of different strength should be used to determine any dependence of resolving time on counting rate. The coincidence rate is given by

$$
\mathrm{M}=\mathrm{B}_{\mathrm{c}}+2 \tau \mathrm{N}_{1} \mathrm{~N}_{2}
$$

where $B_{c}$ is the independently measured cosmic xay background coincidence rate, and $N_{1}$ and $N_{2}$ are the singles counting rates.

Since it is desirable to keep the chance rate below the true coincidence rate, a limitation is imposed on the source strength. For $C_{R}$ to be less than $N_{C}$, it is necessary that $2 \tau M$ be less than unity.

Background activity must be subtracted from the observed counting rates. In the gamma counter the background is determined simply from a measurement using a blank sample mounting. (For sources of high beta activity, bremsstrahlung in the beta absorber may also contribute to the gamma background.) The beta counter, in addition to regis tering normal background activity, also records some gamma counts from the source. [In a more complex decay scheme, these gamma counts may give rise to $\gamma-\gamma$ coincidences.] The background for the beta (and coincidence) channel is determined with an absorber (usually aluminum) shielding the beta counter so as to stop all betas from the source. The effect of the absorber on the gamma counting efficiency in the beta counter must be determined empirically. With beta counters whose gamma counting efficiency is low, the correction will be small.

Internal conversion of the gamma ray may take place. For the simple decay scheme under consideration (one beta followed by a single gamma) such internal conversion will contribute electron counts to the beta counter and $\mathrm{N}_{\beta}$ will be given by

$$
\mathbf{N}_{\beta}=\mathbf{N}\left(\epsilon_{\beta}+a \epsilon_{e}-a \epsilon_{\beta} \epsilon_{e}\right) \quad \text { s }
$$

where a is the fraction of total disintegrations leading to conversion electrons $\left(i, i_{,} a=N_{e} / N_{\beta}\right)$ and is related to the internal conver sion coefficient. 
$\alpha$, by $a=\alpha /(1+\alpha)$, and $\epsilon_{\mathrm{e}}$ is the efficiency of the beta counter for the counting of conversion electrons. The last term, giving $\beta-e$ coincidences can often be neglected if $\epsilon_{\beta}$ and $\epsilon_{e}$ are not too high. Likewise,

$$
\mathbf{N}_{\gamma}=\mathbf{N}(1-\mathbf{a}) \epsilon_{\gamma}
$$

and

$$
\mathbf{N}_{\mathrm{c}}=\mathbf{N}(1-\mathrm{a}) \epsilon_{\gamma} \epsilon_{\beta}
$$

The latter equation has no term in $\epsilon_{e^{2}}$ since there can be no coincidences between the conversion electron and the converted gamma. Then

$$
\frac{N_{\beta} N \gamma}{N_{c}}=N\left(1+\frac{a \epsilon_{e}}{\epsilon_{\beta}}-a \epsilon_{e}\right)
$$

where the last term is negligible if $\epsilon_{\beta}<<1$. It is possible to discriminate against the conversion electrons in the beta counter by the use of appropriate absorbers when the energy of the lectrons is lower than that of the betas. High energy conversion electrons arise from the conversion of high energy gammas, and in such cases a is generally small, giving only a small contribution to the beta counts.

If the beta and gamma have a directional cox relation in the decay process, this must be known so that the observed coincidence rate for a given orientation of sample and counters may be properly interpreted. The use of high geometry in at least one detector tends to minimize effects of angular cor relation.

Dead time losses in the counters contribute errors in $\mathbb{N}$ which are generally small (the order of $1-2 \%$ ). If pulse height selection is employed as indicated in the schematic diagram (Figure 13), so that every pulse en tering the coincidence circuit will also register in one of the singles chan nels, any losses which occurred at the detectors may be considered as part of the detection efficiencies. When the dead times appropriate to the singles channels are the same as those for coincidence registration (i.e., when the coincidence resolving time, $2 T$, is negligible with respect to the dead times), the dead time corrections cancel in the determination of $\mathbf{N}$. However, when the coincidence resolving time is comparable to the dead times of the singles channels, this cancellation is no longer exact. (77)

Statistical errors must be applied to the data in order to estimate the accuracy. If careful measurements are made, the coincidence method for determining disintegration rates of nuclides having simple decay schemes may be expected to yield results having an error of $1-2 \%$.

When complex decay schemes are involved, the treatment presented above must be extended. For example, if a single beta is followed by more than one gamma ray, the gamma counting efficiency is the sum of the 
counting fficiencies of the individual gammas with a small correction for $\gamma=\gamma$ coincidences. If both gammas are converted, their conversion coefficients must be known and applied in the equations for $N_{\beta}, N_{\gamma}$, and $\mathbb{N}_{c}$. When more than one beta ray is emitted, a series of coincidence measurements are made with varying absorber thicknesses in front of the beta counter. A coincidence absorption curve is obtained which may be extrapolated to zero total absorber, and the disintegration rate determined from a knowledge of the beta branching ratios. Alternatively, energy discrimination in either counter may be effected by the use of pulse heightanalyzers. Other combinations of coincidence measurements ( $\operatorname{such}$ as $\gamma-\gamma, \gamma-e^{-}$, or $\beta-e^{-)}$may also be employed in certain cases.

Liquid Beta Counting

In general, liquid counting is employed for relative measurements of rather energetic betas. As in solid counting, the conditions of counting must be reproduced accurately for meaningful results. The problems of solution counting, such as the effects of type and energy of the radiation, geometrical arrangement, wall thickness of the counting tube, and the density and atomic number of the medium, are analogous to those encountered in solid sample counting. Experimental data on these effects in liquids, however, are not very extensive. Some of the problems are reviewed by Cook and Duncan, (78) Solomon and Estes, (79) Chiang and Willard, (80) Walton, (81) and Friedman and Hume. (82)

If absolute counting of liquids is necessary, it is recommended that the counter be standardized with a source of known disintegration rate.

Beta Scintillation Counting

Beta particles may be conveniently detected with scintillating crys tals, such as anthracene, or with plastic scintillators. The problems associated with the samples themselves are identical to those discussed under Geiger and proportional counting. Scintillation detectors offer the same advantages as proportional counters in the ability to discriminate between beta rays of different energy and in having small resolving time losses. In addition, they may be used for beta rays of higher energy than proportional counters. In the low energy region, however, proportional counters give somewhat better resolution.

Liquid scintillators may also be used and are particularly suitable for low energy radiations and as absolute $4 \pi$ detectors when the source is dissolved in the scintillating solution. Since the latter are usually prepared using organic solvents, suitable compounds of the samples must be prepared that will be soluble in the medium and not absorb or otherwise quench the light output of the scintillator. The dimensions of the scintillator should be large compared to the range of the radiation to minimize losses at the edges. 
Backgrounds should be determined using a blank scintillator solution containing a nonradioactive compound isotopic with the sample to be measured. It is also important to be able to reproduce the conditions which may affect phototube noise. Beta rays of very low energy may produce pulses which are masked by the phototube noise pulses, and special techniques such as cooling or coincidence counting may be necessary to reduce the phototube background. 


\section{ALPHA COUNTING}

The extremely short range of alpha particles in matter necessitates the use of detectors having thin windows or provisions for the introduction of samples into the active volume (internal counters). For general alpha assay work, pulse ionization chambers or proportional counters have proven most satisfactory. Current-measuring devices have a number of disadvantages, such as the dependence of ionization current on the alpha energy and the sample thickness, the lack of distinction between the ionization due to alphas and that due to $\beta, \gamma$, or cosmic radiation, and the inability to measure very weak sources. In pulse chambers, one alpha will give rise to a single pulse over a wide range of energies; sample self-absorption is not as important, since a pulse may be detected even after a large fraction of the range has been expended. An alpha pulse can be discriminated from $\beta, \gamma$, or cosmic ray pulses, and extremely low backgrounds ( $<1$ count per minute) can be attained, permitting the accurate measurement of very weak samples.

In general, the same considerations discussed in the case of the determination of beta disintegration rates apply to alpha determinations with quantitative rather than qualitative differences. The counting yield is again dependent on such factors as geometry, scattering, absorption, and detection probability.

Geometry factor. Two geometry factors are generally used: "50\%" (or $2 \pi)$ and "low" $(<1 \%)$. In the $50 \%$ chamber the source is placed inside the chamber and, since half the particles are emitted away from the plate, the relative solid angle is 0.5 . In the low-geometry counter the source is placed at a known distance from an aperture that opens into the chamber. The relative solid angle is calculated from the known dimensions of the system (see Appendix I).

Scattering. Since alpha particles do not scatter easily, the scattering factor may be considered as unity, even in low geometry chambers. However, backscattering from the sample plate does occur and must be corrected for in $50 \%$ chambers.

Backscattering. Not much experimental data have been accumulated on the variation of alpha backscattering with the alpha energy and atomic number of the scatterer. However, it is known qualitatively that backscattexing increases with increasing atomic number $Z$ and with decreasing alpha energy. For the most commonly used sample plates (Pt)

*This section is based largely on the excellent and comprehensive discussion of alpha assay by A. H. Jaffey. (3) 
the backscattering factor has been determinedas 1.04 in a $50 \%$ chamber $(83,84)$ This result is in good agreement with theoretical predictions. (85) In lowgeometry chambers, the backscattexing effect is negligible, and this instrument is useful as an absolute geometry standard.

Absorption. In alpha assay work, it is the general practice to count under such conditions that the effect of external absorbers is negligible. Thus, internal counters or low-geometry chambers having a total absorber (path in gas plus window) equal to less than $90 \%$ of the alpha range are used.

For some measurements not requiring high precision it is possible to use a foil absorption technique to separate the contributions of mixtures of alphas having different ranges. The sample is mounted in a $50 \%$ geometry chamber and the counting rate is measured as a function of the absorber thickness placed over the sample. The alphas are absorbed linearly, and, the "peeling-off" process is used to determine the contributions of the individual components (Figure 14). Thin, smooth samples are necessary for the application of the method, since excessive selfabsorption in the sample results in a nonlinear absorption curve which might erroneously be interpreted as indicating more than one alpha (Figure 15). Other methods, e.g., the use of a differential range chamber(3) or pulse analysis, may also be utilized for the purpose of resolving a multi-component alpha source, but they require special instrumentation.

Self-absorption. $(3,111-116)$ If visible amounts of solid are present in an alpha sample, los ses in counting rate in a $50 \%$ geometry chamber may be expected due to selfabsorption. In a smoothly spread sample, the fraction, E, of alphas lost by self $\rightarrow$ absorption has been calculated $(86)$ to be

$$
F=\frac{1}{2} t / R
$$

where $t$ is the sample thickness and $R$ is the effective range of the alpha particle (i.e., the maximum distance an alpha particle can travel through the source material and still register a pulse in the chamber). $R$ is alo ways less than the true range. Since it is difficult to prepare uniformly spread samples the effect of sample thickness is generally larger than calculated.

Scratches in the surface of the mounting plate may also cause losses in counting rate due to absorption of alphas emitted at low angles from the part of the sample deposited in the scratch.

In low geometry chambers, only those alphas emitted nearly vertically are effective and, hence, if the sample thickness (plus gas and window thickness) is less than the range of the alpha no appreciable losses will occur. Clumping of the sample will usually make the effective thick ness larger than the average thickness of the source. 


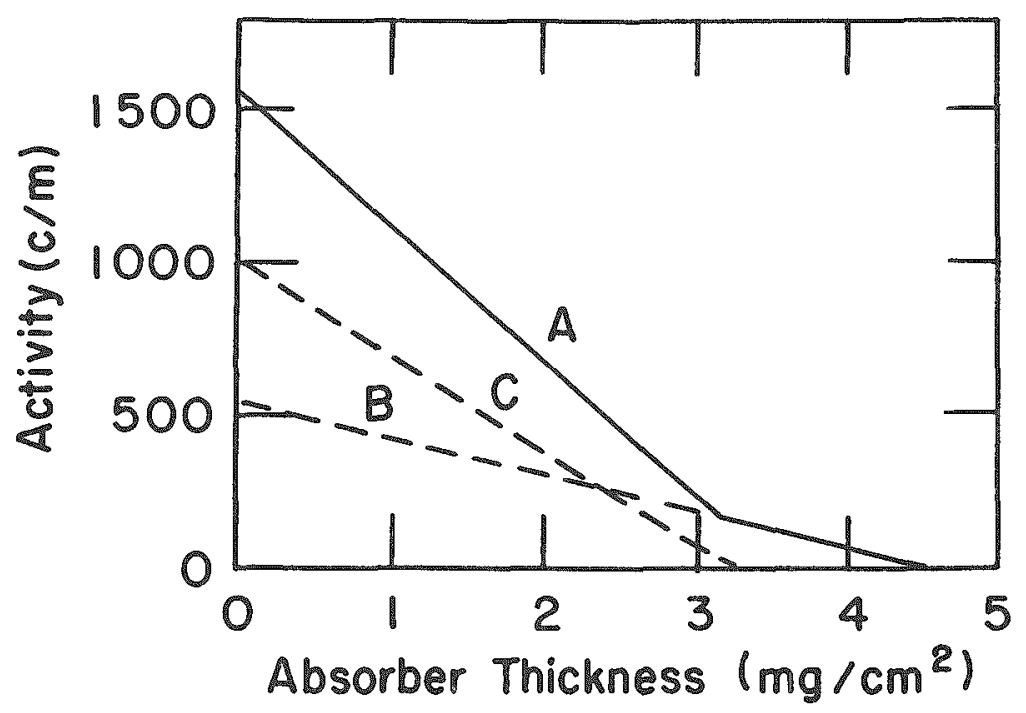

Figure 11

Resolution of a complex curve (range measurement by foil absorption in a 50\% geometry pulse chamber) inco two alphaemitting components with extrapolated ranges of 3.18 and $4.45 \mathrm{mg}$; $\mathrm{cm}^{2}$, respectively.

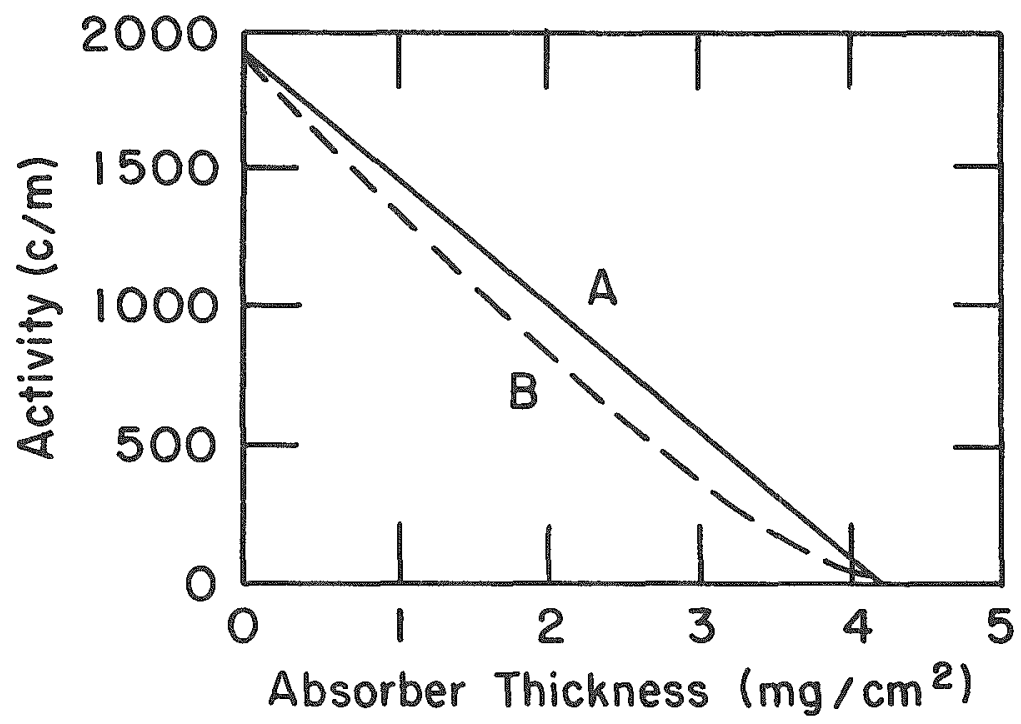

Figure 15

Effect of excessive self-absorption due to sample thickness. Curve A shows the type of curvedetermined from a thin smooth sample. Curve $B$ shows the type of curve determined from a sample having the same type of radioactivity and the same counting rate with no absorber, but with an excessive amount of foreign material, e.g., carrier salt. 
Selfabsorption correction curves may be determined by methods analogous to those employed in the case of beta particles. In the determination of such self-absorption curves it should be realized that, as the thickness of sample increases, the plateau changes and it is advisable to extrapolate the plateau for each sample to zero pulse height for comparison.

Plateaus. Although a knowledge of the proper operating conditions for the detecting instrument is assumed for the purposes of this report, a discussion of the proper operating voltage in relation to the sample properties is pertinent. Thus, it may not always be desirable to use a counter at the flattest portion of its plateau. For the comparison of samples having various degrees of energy degradation (due, e.g., to different self-absorption in the sources) it may be desirable to count far up on the plateau where the degraded pulses will register. For thin samples on the same backing, reproducible counting can be obtained at any point along the plateau, although it is generally desirable to operate a short distance above the knee of the plateau. In internal proportional counters the counting efficiency may depend on the sample area for large samples when operating just above the plateau knee, and it is advisable to keep the samples confined to areas $1 \mathrm{~cm}$ in diameter or less.

Beta pulse pile-up. The proportional alpha counter may be used to count alpha particles in the presence of betas, since the pulse heights produced by the latter are much smaller and may be discriminated against by appropriate biasing. However, when the number of beta particles becomes great enough so that they pile up to produce pulses of sufficient magnitude to be registered, the alpha plateau is shortened and the input threshold of the instrument affected such that a net reduction in alpha counting rate may be observed.(87) The magnitude of the reduction depends on the operating voltage, beta intensity, beta energy, alpha pulse amplitude distribution, input amplitude threshold, and individual instrument circuit characteristics. For a beta intensity of $3 \times 10^{7}$ disintegrations per minute (dpm) the reduction is about $0.5 \%$, and for $4 \times 10^{8} \mathrm{dpm}$, it is about $2 \%$. With sufficient beta activity, the plateau disappears and alpha counting is impossible.

Counting standards. The reproducibility of counting efficiency is normally checked by the use of alpha-counting standards. If it is desired to adjust the external ox internal gain so that a previous counting rate of the standard is reproduced, a thick sample should be used as a standard. In such a sample a wide and continuous distribution of pulse heights is produced and, since essentially no plateau exists, the counting rate is very sensitive to small variations in gain and may be accurately and easily reproduced. For counting a series of similar samples, over a period of time, it is usual to measure ratios of the counting rates of sample and a standard. These ratios are generally more reproducible than are the individual counting rates. 
Resolutior losses. The corrections for coincidence or resolution losses are made as discussed in Appendix II The low geometry counter. is suggested for very active samples whe we the resolution loss correction in a $50 \%$ chamber is too large to be made accurately.

Absolute counting. $(3,117)$ Absolute disintegration rates may be de. termined in $50 \%$ chambers when the backscattering correction is known and selfoabsorption in the source is negligible. The counting efficiency, how ever, w1 $1^{1}$ vary along the plateau because of the slope resulting from the backscattering effect and variatiors of internal amplification for tracks in differert parts of the chamber These effects limit the accuracy of such absolute determinations and introduce errors of the order of $1 \%$.

Lowr geometry counters avoid these effects and very accurate dis untegration rate determinations can be made providing the geometry is accurately defined and calculated or the counter efficiency is calibrated with a source of known disintegration rate.

Relative counting. For many purposes the relative counting rates of sources is sufficiert. It is ther onlv necessary to reproduce the count ing conditiors (such as barkscattering and self-absorption) by mounting the samples idertira.11y It is dufficult to reproduce self-absorption accurately except by making it verv small. For sample thicknesses of the order of $0 \mathrm{Lmg} / \mathrm{cm}^{3}$ Lt is usually possable to spread the samples sufficiently uniformly to attal reproducibiluties to within a few per cent 
GAMMA COUNTING

Relative Counting

The high penetrability of gamma radiation in matter permits some simplification of relative counting techniques. The problems of absorption and sample thickness, which are extremely important in alpha and beta counting, are minimized in gamma counting. Thus, relative gamma in tensity measurements are particularly useful for samples which differ in chemical composition or size and for liquid samples. Of course, in measm uring gamma radiation, sufficient absorber must be placed in front of the detector to screen any accompanying beta particles.

It is sometimes difficult to maintain a constant geometric relationship between source and detector when solid samples of different size are being compared. Such comparisons may be readily made in solution, however, using, e.g., a high pressure ionization chamber detector.(88)

Absolute Gamma Counting

In the determination of absolute intensities of gamma radiation the major problem is a knowledge of the intrinsic efficiency of the detector. This efficiency is dependent on the energy of the gamma ray and the nature of the material of which the detector is made. The ordinary brass walled, argon filled, end-window Geiger counters have an efficiency of about $1 \%$ per Mev of gamma energy. This relationship is quite linear above $0.1 \mathrm{Mev}$. Below this energy the efficiency decreases less rapidly with decreasing energy and finally rises again due to significant absorption in the gas filling. Many devices have been used to increase the efficiency of detectors for gamma radiation, e.g., multiple chamber counters, counters lined with materials of high atomic number, high-pressure ion-chambers, and scin-m tillation detectors.

Any of these detectors may be calibrated with sources of known dis integration rate, and an efficiency curve as a function of gamma ray energy may be determined. The decay schemes of the standards used must, of course, be known, so that the number of $\gamma$ rays accompanying each disintegration may be calculated. The disintegration rate may be determined using the methods outlined in the section on beta counting. The intensity of gamma radiation of known energy in a source is then determined from the counting rate of the sample and the efficiency as read from the calibration curve.

In many instances, two or more gamma rays are involved in a decay process. If the energies are sufficiently different, the relative contributions may be determined from an absorption curve (usually in lead). The logarithm of the activity is plotted against the thickness of absorber. Since gamma radiation is exponentially absorbed, each component will exhibit a half-thickness characteristic of its energy. The resolution of this method 
is rather poor, however, and, in general, the energies must differ by about a factor of two for a successful analysis of the curve. (The resolution is somewhat better in the region of a few hundred kev where the absorption coefficient is a more sensitive function of the energy.) For energies which do not differ sufficiently, a single exponential curve with a half-thickness characteristic of the average energy of the components is obtained.

Experimental verification of theoretical absorption coefficients may be obtained under conditions of so-called "good" geometry, i.e., collimated beams with large distances separating source, absorber, and detector so that secondary radiation is not recorded.(89) Under the usual laboratory counting conditions, "good" geometry is rarely attained, so that secondaries and scattered radiation may be detected. The effect on absoxption curves is to alter the strictly exponential character of the absorption and to introduce curvature which may be erroneously interpreted as an additional component. The exact nature of the effect is dependent on the particular conditions involved. A satisfactory technique is to place one lead absorber $\left(\sim 1 \mathrm{gm} / \mathrm{cm}^{2}\right.$ ) adjacent to the detector window. The position of additional absorbers between the sample and the first absorber is not critical. Such an arrangement results in exponential absorption, although the half-thicknesses observed are somewhat larger than theoretical. The efficiency curve should be determined with the same absorber in position adjacent to the detector if absolute intensities are to be determined.

Scintillation Counting - Pulse Height Analysis

Scintillation counting has proved extremely valuable for $\gamma$ counting, (90) and it is rapidly replacing other techniques. Using thallium-activated Nal crystals, detection efficiencies approaching $100 \%$ may be achieved. The scintillation detector may be calibrated using standard sources and may be used for integral or differential counting. The former is perhaps somewhat simpler and is not so subject to the electronic difficulties associated with pulse height analysis. It is recommended for relative counting or for absolute intensity measurements of samples known to emit a single gamma or X ray. (Multiple gammas may be separated by absorption as described above, with the same limitation on resolution.)

For the determination of energies and absolute intensities of gamma rays in complex systems, the scintillation detector may be coupled with a differential pulse-height analyzer. Such a combination is generally referred to as a "scintillation spectrometer." (91) Such an instrument may be conveniently used for disintegration $x$ ate determinations $(96,97,100)$ and as a means of identifying radioactive species. $(100,101)$ It is convenient to calibrate the scintillation spectrometer in terms of the full-energy-peak counting efficiency to avoid the difficulty of separating the overlapping pulse-height distributions from two or more gammas of different energy. Curves of the full-energy-peak counting efficiency as a function of energy 
may be derived from the total counting efficiency data of Bell, et al. (92) for a $1 \frac{1}{2}-i n$. diameter by 1 -in. high $\mathrm{NaI}(\mathrm{T} 1)$ crystal and the ratio of integrated photo peak to total counts measured by Heath, et al.(93) and McGowan. (94) This has been done by Kalkstein and Hollander (95) and Heath and Schroeder.(96) Their curves are reproduced in Figures 16-19. The properties of some useful standards for calibration of systems not adequately represented by the curves of Figures $16-19$ are given in Table I. $(97,98)$ Disintegration rates for these nuclides may be determined by $4 \pi$ or coincidence-counting techniques.

Table I

USEFUL STANDARD SOIRCES FOR $y$-INTENSITY CALIBRATIONS

\begin{tabular}{|c|c|c|c|c|c|}
\hline Nuclide & Ha $1 \mathrm{f}-\mathrm{Life}$ & $\begin{array}{c}\gamma \text { Energy } \\
\text { (Mev) }\end{array}$ & $\begin{array}{l}\text { No. of Gammas } \\
\text { per } \\
\text { Disintegration }\end{array}$ & $\begin{array}{c}\beta \text { Energy } \\
(\text { Mev })\end{array}$ & $\begin{array}{c}\text { Method of } \\
\text { Detn. of } \\
\text { Disintegration } \\
\text { Rate }\end{array}$ \\
\hline $\mathrm{Na}^{22}$ & $2.60 \mathrm{y}$ & $\begin{array}{l}1.277 \\
0.511\end{array}$ & $\begin{array}{l}1.11^{\mathrm{a}} \\
2.00^{2}\end{array}$ & 0.542 & $\begin{array}{l}4 \pi \text { counting, } \\
\beta-\gamma \text { coinc. }\end{array}$ \\
\hline $\mathrm{Na}^{24}$ & $15.0 \mathrm{~h}$ & $\begin{array}{l}1.38 \\
2.76\end{array}$ & $\begin{array}{l}1.00 \\
1.00\end{array}$ & 1.39 & $\begin{array}{l}4 \pi \text { counting, } \\
\beta-\gamma \text { coinc. }\end{array}$ \\
\hline $\mathrm{Sc}^{46}$ & $85 \mathrm{~d}$ & $\begin{array}{l}0.89 \\
1.12\end{array}$ & $\begin{array}{l}0.995 \\
1.00\end{array}$ & 0.36 & $\begin{array}{l}4 \pi \text { counting, } \\
\beta-\gamma \text { coinc. }\end{array}$ \\
\hline $\mathrm{Co}^{60}$ & $5.27 y$ & $\begin{array}{l}1.332 \\
1.172\end{array}$ & $\begin{array}{l}1.00 \\
1.00\end{array}$ & 0.306 & $\begin{array}{l}4 \pi \text { counting, } \\
\beta-\gamma \text { coinc. }\end{array}$ \\
\hline $\mathrm{Nb}^{95}$ & $35 d$ & 0.745 & 1.00 & 0.160 & $4 \pi, \beta-\gamma$ coinc. \\
\hline $1^{131}$ & $8.14 d$ & 0.364 & 0.80 & 0.815 & $4 \pi$ \\
\hline $\mathrm{Au}^{198}$ & $2.69 d$ & 0.412 & 0.957 & 0.963 & $4 \pi$ \\
\hline $\mathrm{Hg}^{203}$ & $47 d$ & 0.279 & 0.81 & 0.208 & $4 \pi, \beta-\gamma$ coinc. \\
\hline $\mathbb{T 1}^{208}$ & $3.1 \mathrm{~m}^{\mathrm{b}}$ & 2.62 & 1.00 & 1.79 & $a_{\text {. counting }}$ \\
\hline $\mathrm{Am}^{241}$ & $500 y$ & 0.0596 & 0.357 & $a$ & a-counting \\
\hline
\end{tabular}

Number of gammas per positron.

${ }^{\text {bupported by } 10 \cdot 6 \cdot \mathrm{h} \mathrm{Pb}^{2} 12}$ 


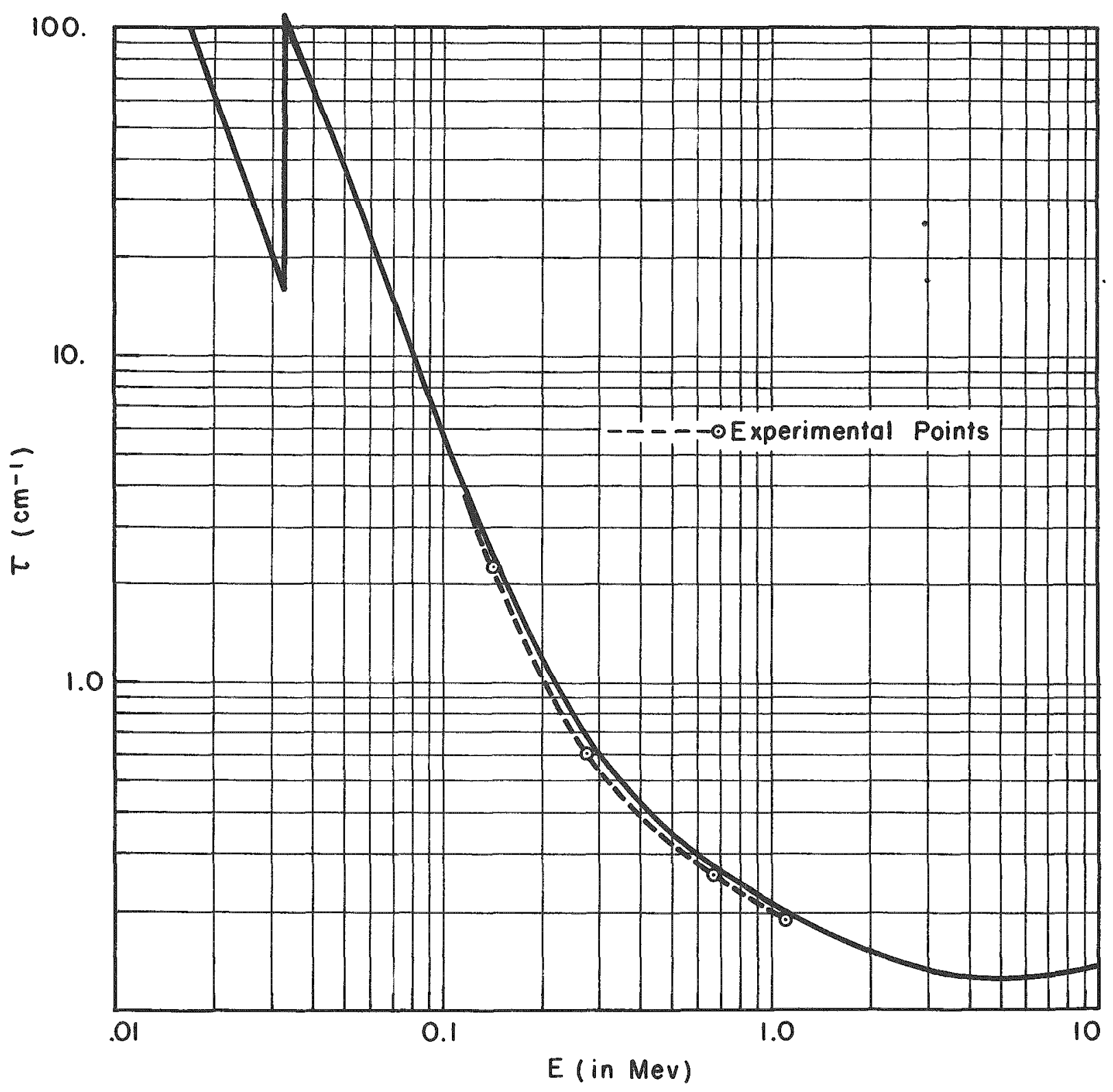

Figure 16

Linear absorption coefficient for $\mathrm{NaI}$ from White, NBS 1003 (using density $=3.667 \mathrm{~g} / \mathrm{cm}^{-3}$ ). 


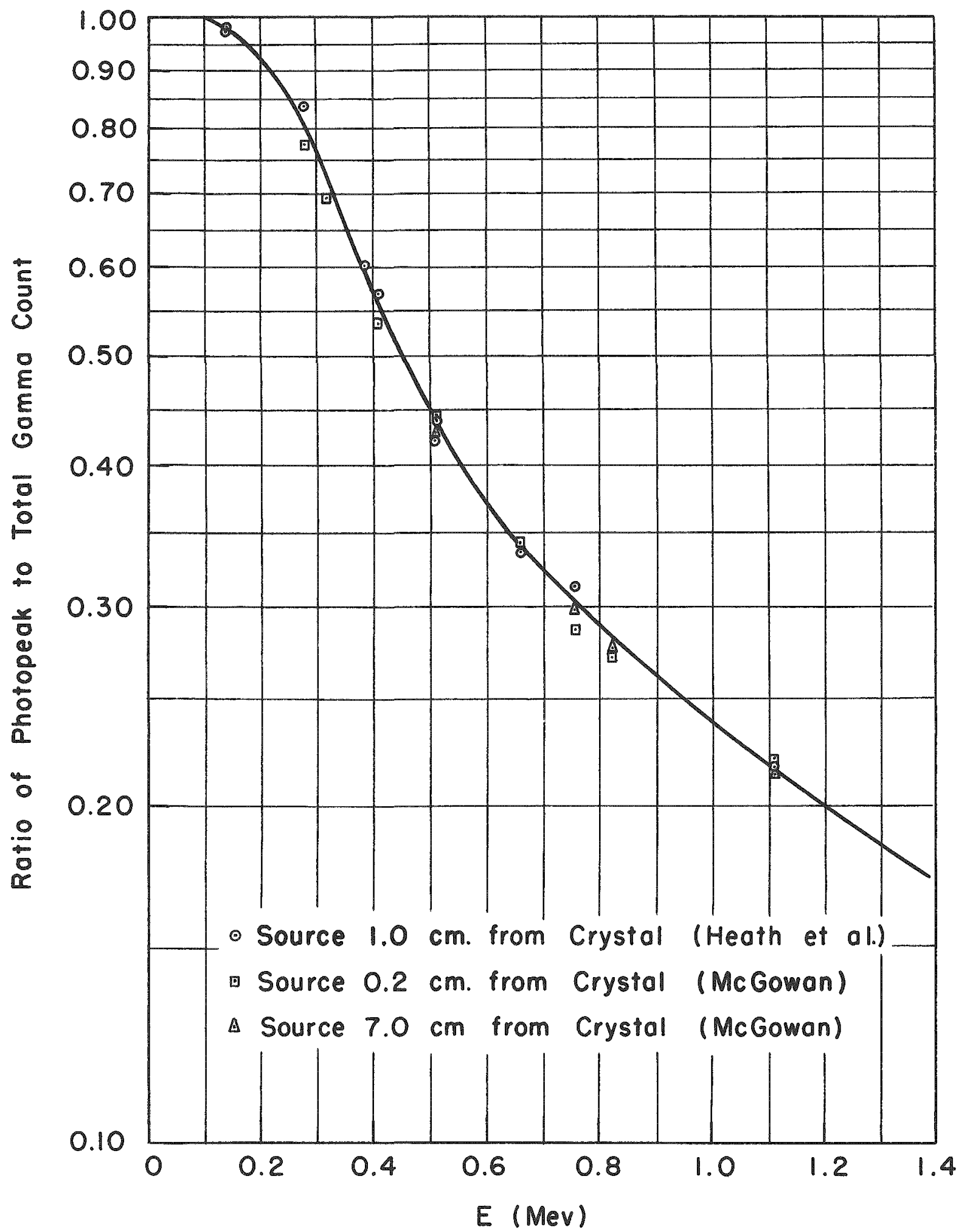

Figure 17

Ratio of integrated photopeak to total gamma counts produced in a NaI crystal $-1 \frac{1}{2}$ in. dia. $x 1$ in. high. 


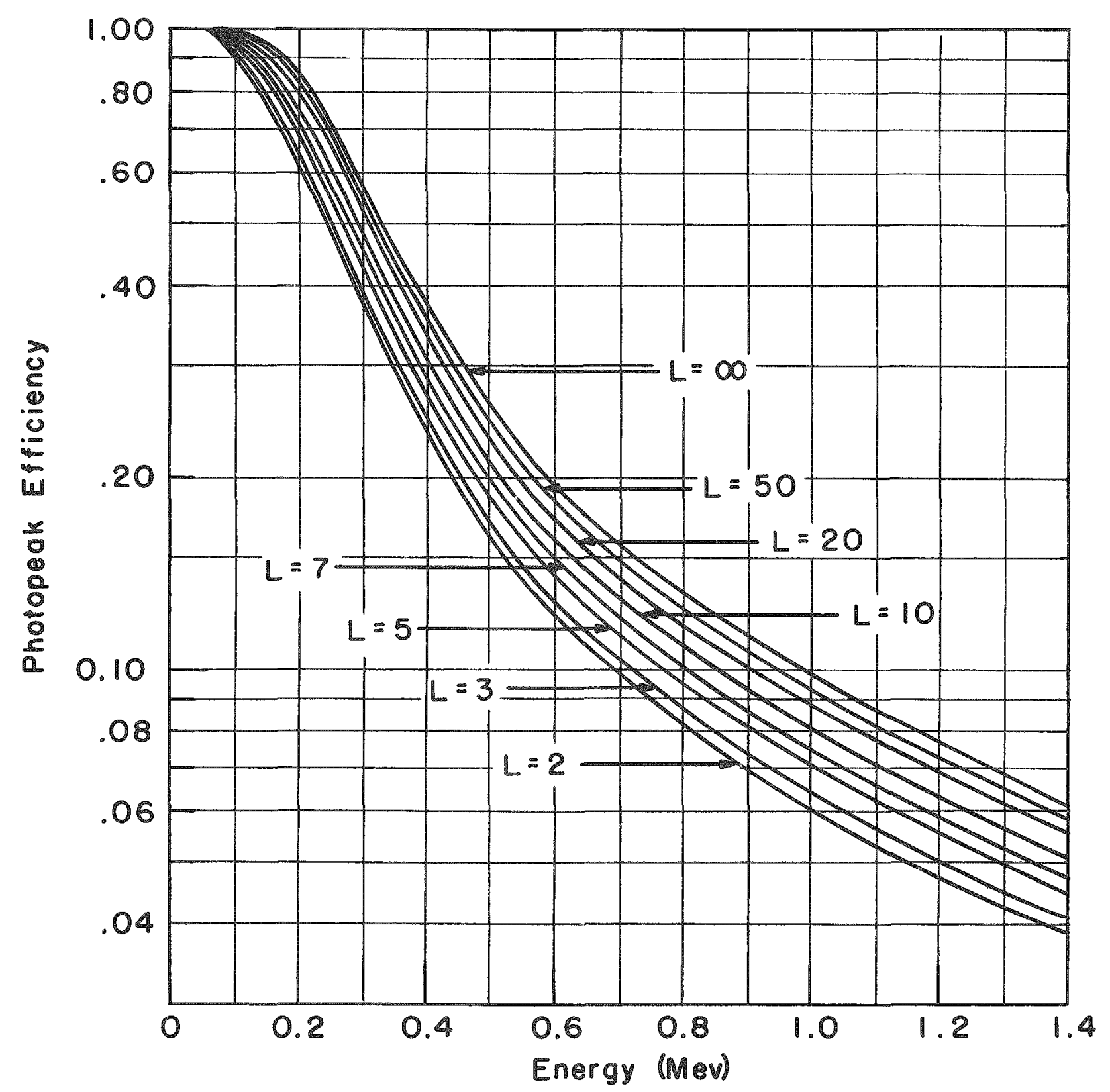

Figure 18

Calculated photopeak efficiency of NaI crystal - $1 \frac{1}{2}$ in. dia. $x 1$ in. high. $L=$ distance in centimeters from source to crystal (source centrally located below crystal). 


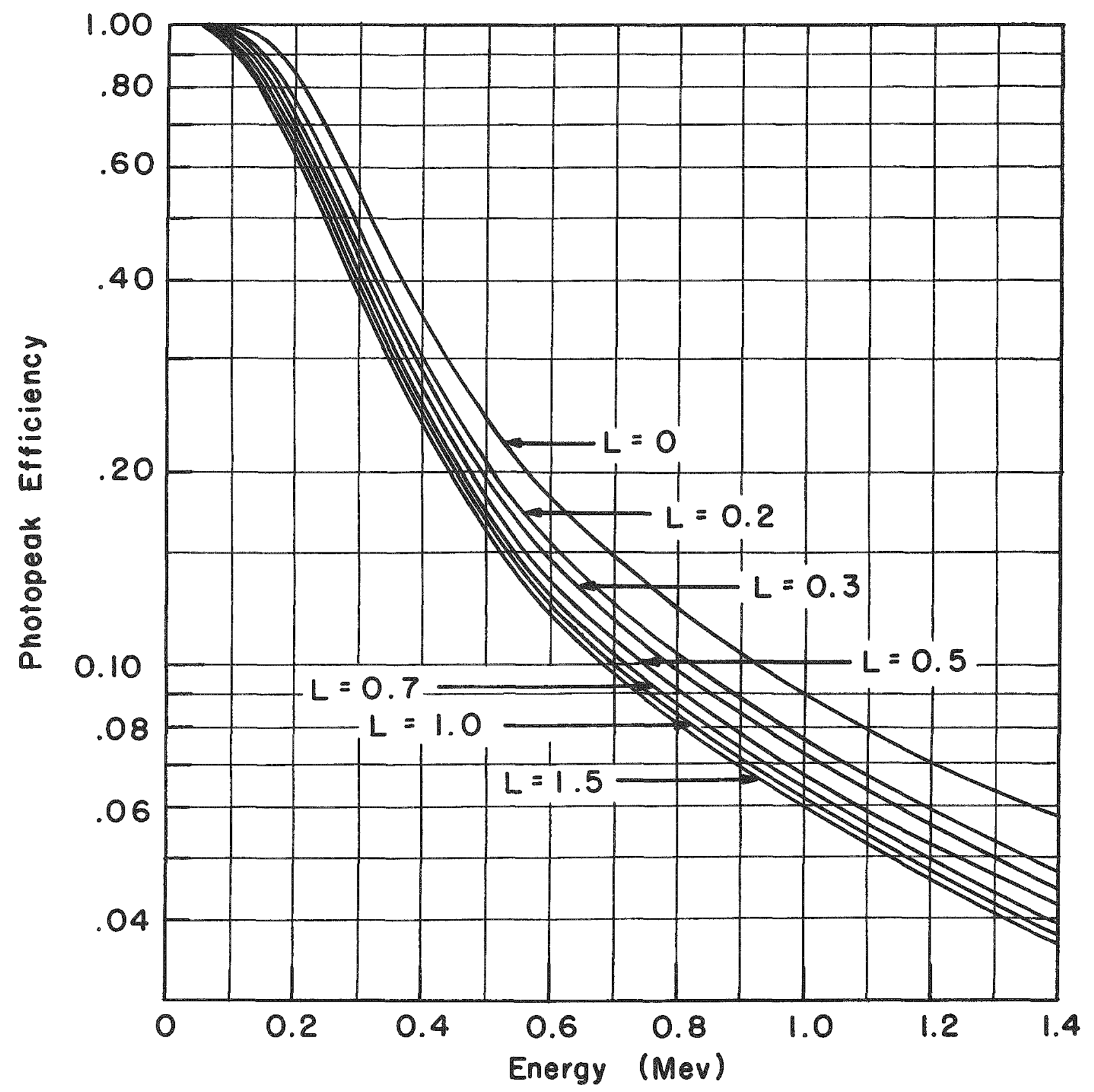

Figure 19

Calculated photopeak efficiency of NaI crystal - $1 \frac{1}{2}$ in. dia. $x 1$ in. high. $L=$ distance in centimeters from source to crystal (source centrally located below crystal). 
Convenient stamdards may not be readily available for calibration, particularly in the low energy region. In the energy region below about $150 \mathrm{kev}$ essentially all of the gamma rays interact by the photoelectric process and the pulse-height distribution exhibits a full-energy ox photo peak. Events occurring near the edges of the crystal may result in the loss of pulses from the full energy peak. To avoid these edge effects, the crystal edges may be screened by the use of a collimator or iris. Under these conditions, the intrinsic efficiency of the crystal is essentially $100 \%$ and the geometric efficiency may be calculated from the dimensions of the system.

Iodine $X$ rays produced near the surface of the crystal may not interact further and may leave the crystal. This loss is observable in the pulse height distribution as an additional peak about $28 \mathrm{kev}$ below the photopeak for incident gamma-ray energies of less than about $150 \mathrm{kev}$ (and greater than $33.2 \mathrm{kev}$, the $\mathrm{K}$-edge of iodine). At higher incident energies, the resolution of scintillation spectrometers is insufficient to distinguish a separate escape-peak. The contribution of pulses in the escape-peak must be added to those in the photo peak to give the total gamma intensity. The escapepeak correction may be determined empirically or calculated. Axel(99) has reviewed the problem and presents calculations of the escape-peak probability as a function of gamma-ray energy based on the approximations of infinitely wide and thick crystals and interaction involving only the photoelectric process. Botk assumptions are shown to be valid for the energy range considered ( $150 \mathrm{kev})$ Figure 20 illustrates the results for the cases of "good" geometry (gammas incident normal to plane of crystal), "intermediate" geometry (crystal subtends a cone of half angle equal to $60^{\circ}$ ), and "poox" geometry (half-angle of cone equal to $90^{\circ}$, i.e., source in direct contact with cystal).

The defined geometry technique is not convenient to apply at higher incident gamma ray energies since the collimating screens become too thick and may introduce scattering effects from their edges. In addition, at higher gamma ray energies, the Compton effect becomes appreciable. and the scattered gammas may undergo multiple processes which contrim bute to pulses in the photo-peak. Such effects are extremely difficult to calculate, and calibration techniques using standard sources are advisable for these energies.

In those cases where two or more gamma rays are emitted within the resolving time of the instrument a peak representing the sum of the gamma-ray energies may be observed. The probability of observing such a peak increases with increasing counting efficiency of the system. For absolute counting rate determination such events must be added to the counting rate of the gamma ray of interest to give the total counting rate. 


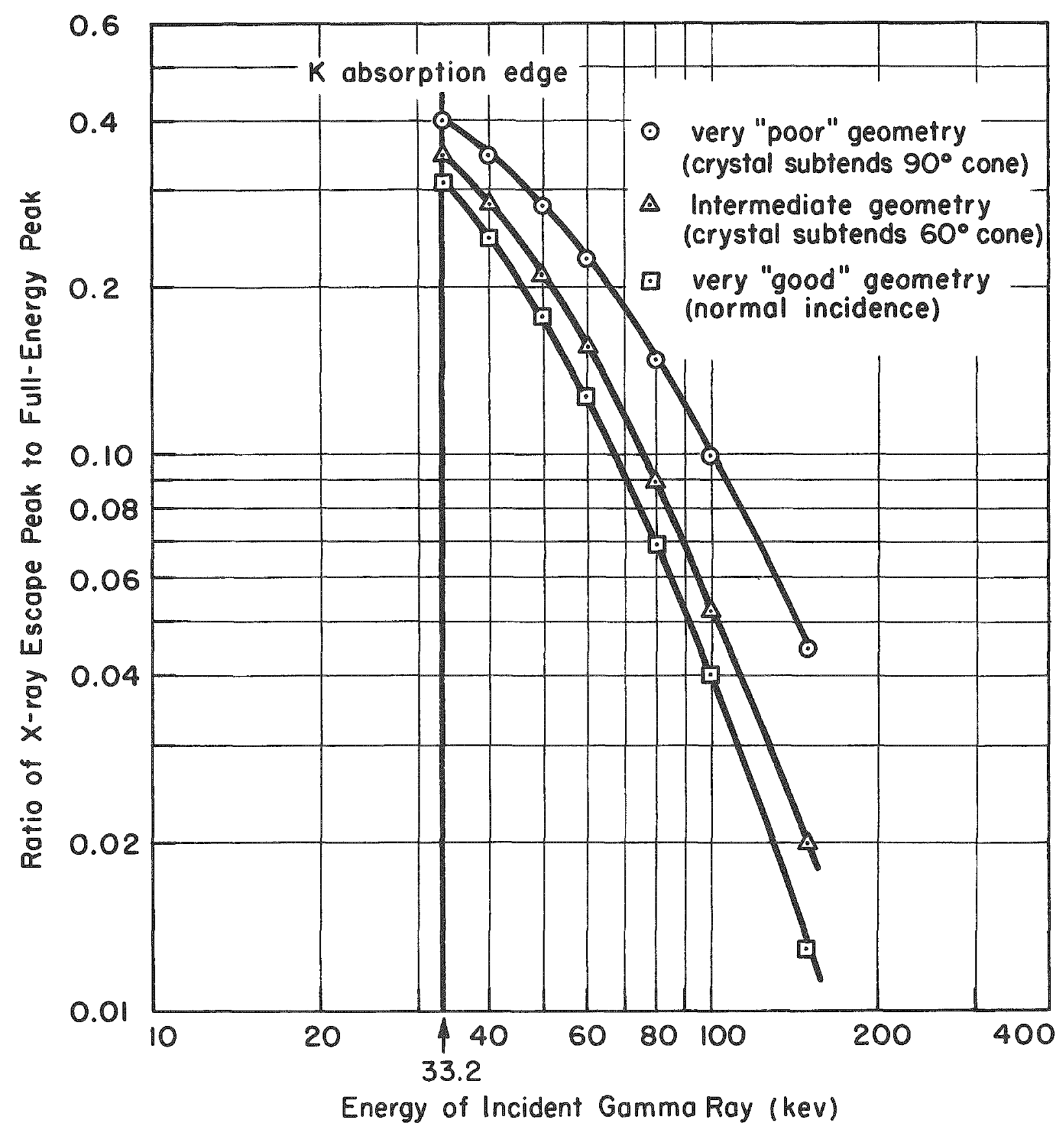

Figure 20

$\mathrm{X}$-ray escape probability for infinitely thick, infinitely wide NaI crystal. (Gamma rays should produce $X$ rays more than $2.5 \mathrm{~mm}$ from the edges or the back surface of crystal.) 


\section{ACKNOWLEDGEMENT}

$I$ should like to express my appreciation to my colleagues, Drs. D. W. Engelkemeir, M. S. Freedman, L. E. Glendenin, A. H. Jaffey, T. B. Novey, and F. T. Porter, for their comments on the manuscript and for their helpful and willing advice during its preparation. 


\section{REFERENCES IN TEXT}

1. A. Berthelot, Compt. rend. 212, 1087 (1941); 1bid., 216, 806 (1943); J. L. Putman, J. Sci. Inst. 26, 198 (1949); M. I. Kalkstein and W. F. Libby, Phys. Rev。, 85,368 (1952), G.I. Mulholland and T.P. Kohman, Phys.Rev., 85, 144 (1952); W. E. Grummitt et al., Can. J. Chem., 34, 206 (1956).

2. A. F. Kovarik, Phil. Mag.20, 849 (1910): J. McClelland, Sci. Trans. Roy. Dublin Soc, IX, Pts. 1 and 2, p. 1 (1906): H. W. Schmidt, Ann. Physik, 23, 671 (1907): H.W.Schmidt, Jahrbuchd.Rad。u. Elek. IV, 4 (20), 451 (1908); J.A. Gray and W. Wilson. Phil. Mag., 20, 870 (1910); A.F. Kovarik and W. Wilson, Phil。Mag.,20,866 (1910); W. Wilson, Proc.Roy.Soc. London, A87, 321 (1912): B.F.J.Schonland, ibid., A104, 235 (1923), Al08, 187 (1925); J.A.Chalmers, Proc.Camb. Phil. Soc. 25 , 331 (1929), 26, 252 (1930): P. B.Wagner, Phys.Rev。 35, 98 (1930); J. L. Saunderson and O.S. Duffenback, Phys。Rev。, 60, 190 (1941).

3. A. H. Jaffey, in The Actunide Elements, G. T. Seaborg and J. J. Katz, eds., Natl. Nucl. Energy Ser., Vol.14A, Div. IV, Chap.16, (McGrawHill, 1954).

4. L. R. Zumwalt, Oak Ridge National Laboratory Report, Mon C-397 (Sept. 1949).

5. B.P.Burtt, Nucleonics. 5. 28 (Aug.1949).

6. T. B. Novey, Rev.Sc1.Instr, 21, 280 (1950).

7. H. O. Anger and C.A. Tobras, Nuclear Sci. Series Prelim. Report No.8, p. 8 (Nat1. Acad. of Sc1. - Natl. Res. Council, Oct. 1950).

8. G. I. Gleason, J. D. Taylor, and D. L. Tabern, Nucleonics 8, 12 (May 1951).

9. J. L. Putman. Brit. J. Radiol., 23, 46 (1950).

10. S. Fried et al., Phys. Rev. 81,741 (1951).

11. G. B. Cook, J.F. Duncan, and M. A. Hewitt, Nucleonics, 8 , 24 (Jan. 1951): G. B. Cook and J.F. Duncan, J. Chem. Soc., S, 369 (1949).

12. J. W. Healy, L. C. Schwendman and R.C. Thorburn, HW-18258 (1950).

13. B. Kalmon Nucleonics, 11,56 (July 1953).

14. J.L. Putman and R.H.Boxall. Brit.J.Rad.20, 190 (1947). 
15. G. G. Manov, Nucl. Sci.Ser. Prelim.Report No. 13 (Natl. Acad. of Sci.- Natl. Res. Council, July 1953); Annual Rev. Nucl. Science 4 , 51 (1954).

16. F. Johnston and J.E. Willard, Science 109, 11 (1949).

17. T. B. Novey and N. Elliott, in Radiochem. Studies: The Fission Products, C. D. Coryell and N. Sugarman, eds, Natl. Nucl. Energy Ser.Vol. 9, Div。IV, Paper 3,(McGraw-Hill, 1951).

18. N. Elliott and E. Shapiro, ibid., Paper 2.

19. M. Deutsch, Phys.Rev.61, 672 (1942): M. Deutsch et al., Phys.Rev. $62,3(1942)$.

20. A. C. Pappas, Mass. Inst. of Tech., Tech. Report No. 63 (Sept. 1953).

21. N. Elliott, D. W. Engelkemeir, and W. Rubinson, in Radiochem. Studies: The Fission Products, C. D. Coryell and N. Sugarman, eds., Nat1. Nucl. Energy Ser. Vol.9, Div. IV, Paper 1, (McGraw-Hill, 1951).

22. S. Eklund, Arkiv Mat. Astron. Fysik, 33A, 50 (1945).

23. D. W. Engelkemeir et al., in Radiochem. Studies: The Fission Products, C. D. Coryell and N. Sugarman, eds. Natl. Nucl. Energy Ser. Vol.9, Div. IV, Paper 5, (McGraw-Hill, 1951).

24. R. E. Cowing and E. DeAmisis, Science, 108, 187 (1948)。

25. L. Yaffe and K. M. Justus, J. Chem. Soc., S, 341 (1949).

26. L. E. Glendenin and A. K. Solomon, Science 112, $623(1950)$.

27. H. H. Seliger, Phys. Rev., 78, 491 (1950); 88, 408 (1952)。

28. J. G. Balfour, J. Sci. Instr., 31, 395 (1954).

29. D. Christian, W.W.Dunning, and D.S.Martin, Ir., Nucleonics 10, 41 (May 1952).

30. G. L. Brownell, Nucleonics, 10, 30 (June 1952).

31. R. H. Muller, Phys. Rev., 93, 891 (1954); see also Chem. and Eng. News 34, 4032 (1956).

32. C. H. Collie, P.F.D. Shaw, and H. J.Gale, Proc. Phys. Soc. A63, 282 (1950); P. F.D. Shaw and C.H. Collie, J. Chem. Soc. 264, 1217 (1949). 
33. D. W. Engelkemeir et al., in Radiochem. Studies: The Fission Products, C. D. Coryell and N. Sugarman, eds., Natl. Nucl. Energy Ser.Vol.9, Div. IV, Paper 4, (McGraw-Hill, 1951).

34. Mme. Percy, J. de Phys. et Rad. 1, 8 (1945).

35. T.E.Banks, Brit. J. Rad., 19, 333 (1946)。

36. M Haissinsky and B. Pullman, J. de Phys. et Rad. 8, 33 (1947).

37. G. Gueben, J. Govaerts, and A. D. M. Stoppani, Ann. Soc. Sci. de Bruxelles, 61, 250 (1947).

38. R. H. Hendricks et al., J. Phys. Chem., 47, 469 (1943).

39. F. C. Henriques et a1., Ind. Eng. Chem., Ana1. Ed, 18, 349, 415 (1946).

40. W. F。 Libby, Anal. Chem.19, 2 (1947).

41. A. K. Solomon, R. G. Gould, and C. B. Anfinsen, Phys.Rev. 72,1097 (1947).

42. E. Broda et al., Proc. Phys.Soc.60, 460 (1948).

43. A. N. Wick, H. M. Barnet, and N. Ackerman, Anal. Chem. 21, 1511 (1949).

44. W. D. Armstrong and J. Schubert, Anal. Chem., 20, 270 (1948).

45. P.E. Yankwich, T.H. Norris, and J.Huston, Anal.Chem.19, 439 (1947): J. Chem. Phys. 14, 131 (1946); P.E. Yankwich and J。W. Weigl, Science 107, 651 (1948): also see P. E. Yankwich in Calvin, et al., "Isotopic Ca rbon" (Wiley, 1949).

46. A. F. Reid, in "Preparation and Measurement of Isotopic Tracers" (J.W.Edwards, Ann Arbor, Mich., 1946).

47. G. K. Schweitzer and B. R. Stein, Nucleonics, 7, 65 (Sept. 1950).

48. C. L. Comar, eta1., Nucleonics 8, 19 (March 1951).

49. W. L. Graf, C. L. Comar, and I. B. Whitney, Nucleonics, 9,22 (Oct.1951).

50. A. H.W.Aten, Jr., Nucleonics 6, 68 (Jan. 1950).

51. W. E. Nervik and P.C. Stevenson, Nucleonics 10, 18 (March 1952). 
52. R. G. Baker and L. Katz, Nucleonics 11, 14 (Feb. 1953).

53. G. N. Walton, J. S. Thompson, and I. F. Croall, Brit. Atomic Energy Res.Est. Report A.E.R.E.C/R 1136 (March 1953)。

54. P. Lerch, Helv. Phys. Acta, 26, 663 (1953); C. Haenny and P. Lerch, ibid., 24, 602 (1951).

55. E. K. Gora and F.C. Hickey, Anal. Chem., 26, 1159 (1954).

56. A. D. Suttle and W. F. Libby, Anal. Chem., 27, 921 (1955).

57. J. G. Cuninghame, M. L. Sizeland, and H. H. Willis, Brit. Atomic Energy Res. Est. Report A.E.R.E.C/R 1646 (July 1955).

58. W. F. Libby, Phys. Rev.46, 196 (1934); W.F. Libby and D. D. Lee, Phys.Rev。 55,245 (1939).

59. See B.D.Pate and L. Yaffe, Can. J.Chem. (a) 33, 15 (1955); (b) 33, $610(1955):$ (c) 33,929 (1955): (d) 33, $1656(1955)$, (e) 34, $265(1956)$.

60. R. A. Clark and G. Failla, Phys.Rev. 75, 328A (1949).

61. R. C. Hawkings, W. F. Merritt and J.H. Craven, Proceedings of Symposium on Maintenance of Standards, Nat1. Phys. Lab., May 1951 (H. M. Stationers Office, London 1952).

62. H. H. Seliger and L. Cavallo, J.Res. Natl. Bur. Stds. 47, 41 (1951); W. B. Mann and H. H. Seliger, ibid., 50, 197 (1953).

63. F.G. Houtermans et al., Z. Physik, 134, 1 (1952); L. Meyer-Schütsmeister, and D. H. Vincent, ibid., 9 (1952), D. B. Smith, Brit. Atomic Energy Res. Est.Report A.E.R.E.I/R 1210 (1953).

64. J. Milsted, Brit. Atomic Energy Res.Est. Report A.E.R.E. C/R 1379 (1954).

65. H. H. Seliger and A. Schwebel, Nucleonics 12, 54 (July 1954).

66. S. C. Brown and W.W. Miller, Rev. Sci. Instr. 18, 496 (1947): B. J. Fontana, J.Am. Chem. Soc. 64, 2503 (1942).

67. A. G. Engelkemeir et al., Phys. Rev. 75, 1825 (1949).

68. R. C. Hawkins, R. F. Hunter, and W.B.Mann, Can. J.Res., 27, 555 (1949). 
69. C. D. Jannay and B. J. Moyer, Rev. Sci. Instr. 19, 667 (1948), W. P. Jesse et al., Phys。Rev., 72, 478 (1947); H. Palevsky, R. K. Swank, and R.Grenchık, Rev.Sci. Instr。18, 298 (1947); J. D. Roberts et al., Anal. Chem.20, 904 (1948); G. L. Brownell and H. S. Lockhart, Nucleonics $10,26($ Feb. 1952).

70. A. G. Engelkemeir and W. F. Libby, Rev. Sci. Instr. 21, 550 (1950).

71. M. L. Eidinoff, "Conference on Absolute $\beta$ Counting," Nucl. Sci. Series Prelim. Report No.8, Paper 9 (Natl. Acad. of Sci.-Natl. Res.Council, Oct. 1950).

72. A. S. Newton et a1., in Radiochem. Studies: The Fission Products, C.D. Coryell and N. Sugarman, eds., Natl. Nucl. Energy Ser.Vol.9B, Div. IV, Paper 145 (McGraw-Hill, 1951); E. J. Hoagland and N. Sugarman, ibid., Papers 146 and 147.

73. F.D. Rosen and W. Davis, Jr., Rev。Sci. Instr.24, 349 (1953).

74. J.V.Dunworth, Rev。Sci。Instr.11, 167 (1940).

75. M。 L。 Wiedenbeck, Phys.Rev。, 72, 974 (1947) M.L.Wiedenbeck and K. Y.Chu, Phys.Rev.72, 1164,1171 (1947).

76. J.Barnothy and M. Forro, Rev.Sci.Instr.22, 415 (1951)。

77. M.S. Freedman et al., ANL-5525, pp. 29-34 (Feb. 1956).

78. G. B. Cook and J.F. Duncan "Modern Radiochemical Practice" (Oxford, 1952).

79. A. I. Solomon and H. D. Estes, Rev. Sci. Instr. 19, 47 (1948).

80. R. S. Chiang and J.E.Willard, Science, 112, 81 (1950).

81. G. N.Walton, Physica, 18, 1280 (1952).

82. A. J. Friedman and D. N. Hume, Science, 112,46 (1950).

83. B. B. Cunningham, A. Ghiorso, and J.C.Bindman, The Transuranium Elements, G.T.Seaborg, J.J.Katz, and W.M. Manning, eds., Natl. Nucl.Energy Ser., Vol.14B, Div.IV, Paper 16.3 (McGraw-Hill, 1949)

84. J.H. Parsons et al., ibid. Paper 16.4.

85. J.A. Crawford, ibid., Paper 16.55.

86. B. B. Cunningham, A. Ghiorso and A. H. Jaffey, ibid., Paper 16.6. $\log ^{2}$ 
87. D. E. Miller and M. B. Leboeuf, Nucleonics, 11, 28 (Apri1, 1953).

88. J. W. Jones and R. T. Overman, USAEC Declassified Document, AECD-2367 (March 20, 1948).

89. C. M. Davisson and R. D. Evans, Rev. Mod. Phys., 24, 79 (1952).

90. P. R. Bell and J. Cassidy, Phys. Rev., 79, 173 (1950).

91. R. Hofstadter and J. A. Macintyre, Nucleonics, 7, 32 (Sept. 1950).

92. P. R. Bell et a1., Oak Ridge National Lab. Phys. Dept. Quarterly Report, ORNL-1415 (1952).

93. R. L. Heath, P. R. Bell and R. C. Davis, ibid.

94. F. K. McGowan, Phys. Rev., 93, 163 (1954).

95. M. I. Kalkstein and J. M. Hollander, University of Calif. Rad. Lab. Report, UCRL-2764 (Oct. 1954).

96. R. L. Heath and F. Schroeder, Phillips Petrol.Co., Atomic Energy Div., Idaho Falls, Idaho Report IDO-16149 (April, 1955).

97. B. Kahn and W. S. Lyon, Nucleonics, 11, 63 (Nov. 1953).

98. D. W. Engelkemeir, private communication (Sept. 1956).

99. P. Axel, Brookhaven Natl. Lab. Report, BNL-271 (Sept. 1953).

100. L. Salmon, Brit. Atomic Energy Res. Est. Report, A.E.R.E.C/M 206 (May, 1954).

101. C. E. Crouthamel and C.E. Johnson, ANL-4924 (Nov. 1952).

102. See, e.g., S. C. Brown, Nucleonics, 3, 50 (Aug. 1948).

103. K. F. Flynn, Argonne Natl. Lab., unpublished results.

104. E. L. Abers, Nucleonics, 3, 43 (Oct. 1948).

105. T. T. Hutchens, et al., Nucleonics, 7, 41 (Sept. 1950).

106. E. P. Steinberg and L. E. Glendenin, Argonne Natl. Lab., unpublished results.

107. C. J. Borkowski and T.H. Handley, ORNL-1056 (April 20, 1951). 
108. D. C. Conway and J. O. Rasmussen, Univ. of Calif. Rad. Lab. Report, UCRL-2075 (January 7, 1953).

109. G. K. Schweitzer, B.R. Stein and J.W. Nehls, J.Phys.Chem.56, 692 (1952).

110. F. C. Larson et a1., Anal. Chem.21, 1206 (1949).

11. R. D. Evans, Phys. Rev., 45 29, 38 (1934).

112. G. D. Finney and R. D. Evans, Phys.Rev., 48, 503 (1935).

113. N. B. Keevil and W. E. Grashem, Can. J.Res., 21A, 21 (1943).

114. H. H. Nogami and P.M. Hurley, Trans.Am. Geophys. Union, 29, 335 (1948).

115. R. D. Evans and C. Goodman, Phys. Rev., 65, 216 (1944).

116. M. Blau and H. Sinason, Science 106, 400 (1947).

117. M. L. Curtis et al., Nucleonics, 13, 38 (May, 1955).

118. T. T. Sugihara, R. L. Wolfgang and W.F.Libby, Rev。Sci.Instr.24, 511 (1953).

119. H. Slätis, in Beta-and Gamma-Ray Spectroscopy, K. Siegbahn, ed., Chapter VIII (II)(Interscience, 1955).

120. A. C. G. Mitchell, ibid., Chapters VII and VIII (I). 


\section{GENERAL REFERENCES ON THE MEASUREMENT}

OF RADIOACTIVITY

Beckerley (Ed.), Annual Reviews of Nuclear Science (Annual Reviews Inc.)

Bleuler and Goldsmith, "Experimental Nucleonics" (Rhinehart, 1952).

Broda, "Advances in Radiochemistry" (Cambridge, 1950).

Calvin et al., "Isotopic Carbon" (Wiley, 1949).

Chemical Inst. of Canada, "Proceedings of Conference on Nuclear Chemistry" (held at McMaster Univ., Hamilton, Ont., May 15-17, 1947).

Chemical Inst. of Canada, "Symposium on Nuclear and Radiochemistry" (held at McGill University, Montreal, Sept. 7-9, 1955).

"Conference on Absolute Beta Counting," Nuclear Science Series Prelim. Report No. 8 (Nat1.Acad。 of Science-Nat1. Res. Council, Oct. 1950).

Cook and Duncan, "Modern Radiochemical Practice" (Oxford, 1952).

Coryell and Sugarman (Eds.) "Radiochemical Studies The Fission Products," Vol. 9, Div. IV, Natl. Nuclear Energy Series (McGraw-Hill, 1951).

Curtiss, "Measurements of Radioactivity," U.S. Dept. of Commerce-Natl. Bureau of Standards circular 476, (1949).

Friedlander and Kennedy, "Nuclear and Radiochemistry" (Wiley, 1955).

Frisch (Ed.) "Progress in Nuclear Physics" (Pergamon, Annually).

Glascock, "Labelled Atoms" (Interscience, 1951).

Graves and Froman (Eds.) "Miscellaneous Physical and Chemical Techniques of the Los Alamos Project," Vol. 3, Div. V, Natl. Nuclear Energy Series, (McGraw-Hill, 1952).

Guest, "Radioisotopes" (Pitman, 1951).

Hahn, "Applied Radiochemistry" (Cornell, 1936).

Hevesy, "Radioactive Indicators" (Interscience, 1948).

Jaffey, Kohman, and Crawford, "A Manual on the Measurement of Radioactivity," USAEC Declassified Doc. MDDC-388 (Aug. 1944). 
Kamen, "Radioactive Tracers in Biology" (Academic Press, 1951).

Kennedy, "Radioactive Atoms and Isotopic Tracers," (Penn. State College, 1952).

Lapp and Andrews, "Nuclear Radiation Physics" (Prentice-Hall, 1954).

Lawrence and Hamilton (Eds.), "Advances in Biological and Medical Physics," Vols. I and II (Academic Press 1948, 1951).

"Nucleonics," (McGraw-Hill, Monthly).

Nucleonics and Analytical Chemistry Symposium, Anal. Chem. 21, 318-368 (1949).

Paneth, "Radioelements as Indicators" (McGraw-Hill, 1928).

Pannell, "Radioactive Measurement Techniques," USAEC Declassified Doc. $A E C D-2270$ (Nov. 13, 1947).

Roddis (Ed.) "Preparation and Measurement of Isotopes and Some of their Medical Aspects" (Suppl., to U.S. Naval Med.Bull., March-Apri1, 1948).

Seaborg and Katz (Eds.) "The Actinide Elements,"Vol. 14A, Div. IV, Nat1. Nuclear Energy Series (McGraw-Hill, 1954).

Seaborg, Katz and Manning (Eds.) "The Transuranium Elements," Vol.14B, Div.IV, Nat1. Nuclear Energy Series (McGraw-Hil1, 1949).

Segre", "Experimental Nuclear Physics" (Wiley, 1953).

Siegbahn (Ed.) "Beta and Gamma-Ray Spectroscopy" (Interscience, 1955).

Siri (Ed.), "Isotopic Tracers and Nuclear Radiations," (McGraw-Hill, 1949).

Wahl and Bonner (Eds.), "Radioactivity Applied to Chemistry" (Wiley, 1951).

Whitehouse and Putman, "Radioactive Isotopes," (Oxford, 1953).

Wilson, Neir, and Reimann (Eds.), "Preparation and Measurement of Isotopic Tracers" (Edwards, 1946).

Yagoda, "Radioactive Measurements with Nuclear Emulsions," (Wiley, 1949). 


\section{APPENDIX I. FORMULAS FOR GEOMETRY CALCULATIONS*}

The "geometry" of the aperture for a point source, $G_{p}$, is defined as the fraction of the total solid angle subtended by the aperture, i.e.,

$$
\mathrm{G}_{\mathrm{p}}=\frac{\Omega_{\mathrm{p}}}{4 \pi}
$$

For the case of a point source on the axis of a circular aperture (see Figure 1),

$$
G_{p^{\prime}}=\frac{1}{2}\left[1-\frac{Z}{D}\right]=\frac{1}{2}\left[\frac{a^{2}}{D(D+Z)}\right] \text {. }
$$

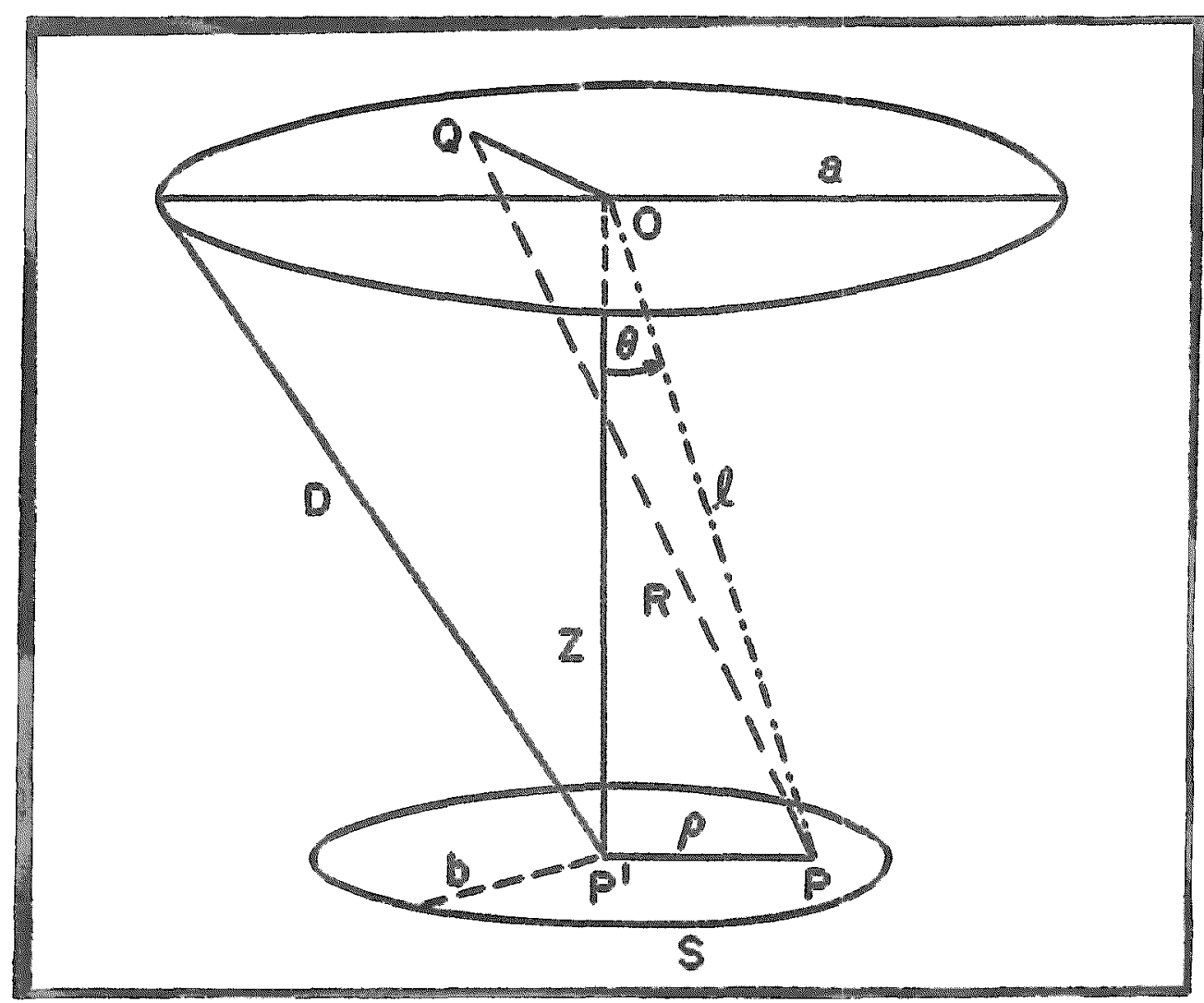

Figure 1

Relation of source and aperture. $O=$ origin, $P Q=R, \quad O P^{\prime}=2, \quad P P^{\prime}=\rho, \quad \cos \gamma=z / R$, $O P=l=\left(Z^{2}+\rho^{2}\right)^{\frac{1}{2}}, z / l=\cos \theta=x, D=\left(z^{2}+a^{2}\right)^{\frac{1}{2}}$.

* For a much more extensive discussion and tables of point source geometries see A. H. Jaffey, Rev. Sci. Instr. 25, 349 (1954). 
For the case of a point source off the axis of a circular aperture, and $\rho / D<1$,

$$
\begin{aligned}
G_{p} & =G_{p^{\prime}-\frac{3}{8} \rho^{2}} \frac{a^{2} Z}{D^{5}}+\frac{15}{32} \rho^{4} \frac{a^{2} z}{D^{9}}\left(z^{2}-\frac{3}{4} a^{2}\right)+\ldots \\
& =G_{p^{\prime}}+\frac{1}{2} \frac{Z}{D} \sum_{n=1}^{\infty} \frac{(-1)^{n}(2 n+1) !}{2^{4 n}(n !)^{2}}\left(\frac{\rho^{2}}{D^{2}}\right)^{n} \sum_{k=0}^{n} m_{k}
\end{aligned}
$$

where

$$
m_{k}=\frac{(-1)^{k+1}(4 n-2 k) !}{(2 n-k) !(2 n-2 k+1) !}\left(\frac{z^{2}}{D^{2}}\right)^{n-k}
$$

and $G_{p^{\prime}}$ is taken for the same value of $Z$ and is given by equation (2).

For the case of a uniformly spread circular source of radius $b$, coaxial with and parallel to the aperture,

$$
G_{S}=G_{p^{\prime}}-\frac{3}{16} b^{2} \frac{a^{2} z}{D^{5}}+\frac{5}{32} b^{4} \frac{a^{2} z}{D^{9}}\left(z^{2}-\frac{3}{4} a^{2}\right)+\ldots
$$

\section{Approximate Formulas}

For small displacements, the geometry of an off-axis point may often be fairly well approximated by $G_{p^{\prime}}$. Likewise, where $b$ is sufficiently small, the geometry of a uniformly spread source may be approximated by $\mathrm{G}_{\mathrm{p}}$. The error introduced in these approximations may be determined from the following:

For the case of small $\rho / D$ or $b / D$, approximately,

$$
G_{p}=G_{p}\left\{1-\frac{3}{4} p^{2}\left[\frac{Z(Z+D)}{D^{4}}\right]\right\}
$$

and

$$
G_{s}=G_{p}\left\{1-\frac{3}{8} b^{2}\left[\frac{Z(Z+D)}{D^{4}}\right]\right\} \text {. }
$$

If $\mathrm{a} / \mathrm{Z}$ is also small,

$$
G_{p}=G_{p^{\prime}}\left(1-\frac{3}{2} \frac{p^{2}}{z^{2}}\right)
$$




\section{APPENDIX II. RESOLUTION LOSS CORRECTIONS IN COUNIING}

The instruments used to detect radioactivity have finite resolving powers, and two events which occur within a short time interval may not be distinguished and recorded separately. Thus, some counts may be missed, the fraction lost increasing with increasing counting rate of the sample. This "resolution loss" (sometimes referred to as "dead-time loss" or "coincidence loss") may be determined in a number of ways: (1) the method of paired sources, (2) measurement of calibrated samples, (3) measurement of multiple aliquots, and (4) measurement of the decay of a sample of known half-life. The most general and most accurate method is that of paired sources. Electronic methods of determining the deadtime may also be used.

\section{The Method of Paired-Sources}

Each of two radioactive sources of approximately equal strength is measured separately and then together. Backgrounds must be subtracted from each measurement and care taken to insure constant geometry factors for the measurements. If resolution loss is occurring, the counting rate of the combined samples will be less than the sum of the individual counting rates. It is advisable to prepare the sources on sturdy material and support them in a reproducible fashion, e.g., as illustrated in Figure 1.

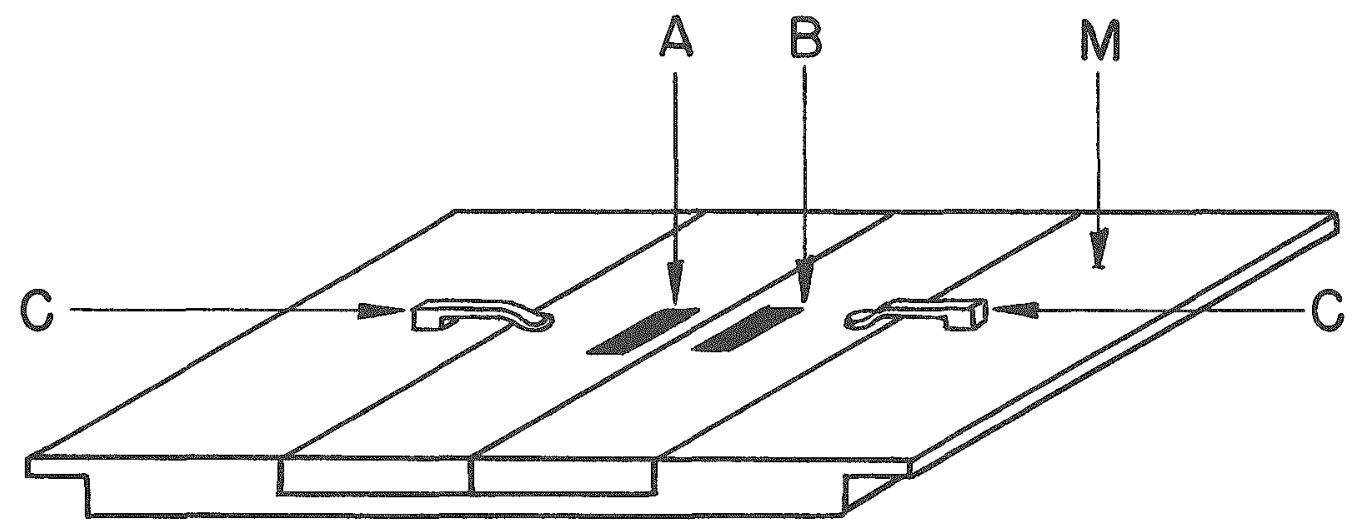

Figure 1

Suggested source-mount for paired-sample technique. M is a metal plate holder which fits shelf of counter assembly, A and $B$ are sources mounted on metal slides, $C$ is a metal clip to hold slides in position. Blank slides are used for backgrounds and to replace either sample during separate counting rate determinations. 
The sequence of measurements necessary for the evaluation of the resolving time is as follows:

a. Place both blanks in position and measure the background, b.

b. Replace one blank by sample $A$ and measure counting rate, $R_{A}$.

c. Replace other blank by sample B (taking care not to disturb position of sample A) and measure the combined counting rates, Rc.

d. Replace sample A by blank (taking care not to disturb the position of sample $B$ ) and measure counting rate $R_{B}$.

The highest statistical accuracy for a given total expenditure of time is obtained when,

$$
t_{A}: t_{B}: t_{C}: t_{b}=\sqrt{R_{A}}: \sqrt{R_{B}}: \sqrt{R_{C}}: \sqrt{b} \approx 1: 1: \sqrt{2}: \sqrt{b / R_{A}} .
$$

It is convenient and desirable to count $R_{A}, R_{B}$ and $R C$ for equal periods of time to obtain the highest statistical accuracy for a given total number of counts. It is also desirable to make $t_{b}$ somewhat greater than indicated by the above ratio.

If a simple equation involving only one parameter relates the true and recorded counting rates, only a single set of measurements involving one pair of samples is necessary. An example of such an equation is

$$
N=R+\tau R^{2}
$$

where $\mathrm{N}$ is the true counting rate, $\mathrm{R}$ is the observed counting rate, and $T$ is the resolution-correction parameter, or dead time.

For correction formulas involving two or more parameters, more data are necessary and may be obtained by measuring a series of paired sources of different counting rates.

A complete treatment of the method of paired sources is given by T. P. Kohman in Seaborg, Katz and Manning, "The Transuranium Elements" N.N.E.S. Vol. 14B, Div. IV, Paper 22.50 (McGraw-Hill, 1949).

\section{Measurement of Calibrated Samples}

If the resolution loss correction has been determined on one counter, a sample or series of samples of different counting rates can be counted with this counter and the true counting rates determined. The samples may then be used to determine the resolution loss corrections for other counters. 
3. Measurement of Multiple Aliquots

A series of samples may be prepared from a stock solution, each differing in activity by a known multiple from the others. These can then be counted and the resolution losses determined from the decrease in the expected counting rate at higher activities. Of course, careful aliquoting and plating of samples is imperative for the application of this technique.

4. Measurement of the Decay of a Sample of Known Half-life

This technique, in principle, is equivalent to method 3. above. The decay of a pure radio-nuclide with accurately known half-life is followed until the activity reaches a level where the resolution losses are negligible and the known half-life is observed. The deviation of the initial portion of the decay curve (high counting rate) from the expected half-life gives a measure of the resolution-losses.

In the application of all of these methods, small differences between large numbers are generally encountered. It is thus necessary that count ing rates be determined with high statistical accuracy. 